\title{
The FOXN3-NEAT1-SIN3A repressor complex promotes progression of hormonally responsive breast cancer
}

\author{
Wanjin Li,, Zihan Zhang, ${ }^{1}$ Xinhua Liu, ${ }^{2,3}$ Xiao Cheng, ${ }^{1}$ Yi Zhang, ${ }^{4}$ Xiao Han, ${ }^{1}$ Yu Zhang, ${ }^{1}$ Shumeng Liu, ${ }^{1}$ Jianguo Yang, ${ }^{1}$ Bosen Xu, \\ Lin He, ${ }^{1}$ Luyang Sun, Jing Liang, ${ }^{1}$ and Yongfeng Shang ${ }^{1,2,3}$ \\ 'Key Laboratory of Carcinogenesis and Translational Research (Ministry of Education), Department of Biochemistry and Molecular Biology, School of Basic Medical Sciences, Peking University Health Science \\ Center, Beijing, China. '2Department of Biochemistry and Molecular Biology, School of Basic Medical Sciences, Tianjin Medical University, Tianjin, China. ${ }^{3}$ Department of Biochemistry and Molecular Biology, \\ School of Basic Medical Sciences, Capital Medical University, Beijing, China. ${ }^{4}$ Center for Cenome Analysis, ABLife Inc., Wuhan, Hubei, China.
}

\begin{abstract}
The pathophysiological function of the forkhead transcription factor FOXN3 remains to be explored. Here we report that FOXN3 is a transcriptional repressor that is physically associated with the SIN3A repressor complex in estrogen receptor-positive (ER+) cells. RNA immunoprecipitation-coupled high-throughput sequencing identified that NEAT1, an estrogen-inducible long noncoding RNA, is required for FOXN3 interactions with the SIN3A complex. ChIP-Seq and deep sequencing of RNA genomic targets revealed that the FOXN3-NEAT1-SIN3A complex represses genes including GATA3 that are critically involved in epithelial-to-mesenchymal transition (EMT). We demonstrated that the FOXN3-NEAT1-SIN3A complex promotes EMT and invasion of breast cancer cells in vitro as well as dissemination and metastasis of breast cancer in vivo. Interestingly, the FOXN3-NEAT1-SIN3A complex transrepresses ER itself, forming a negative-feedback loop in transcription regulation. Elevation of both FOXN3 and NEAT1 expression during breast cancer progression corresponded to diminished GATA3 expression, and high levels of FOXN3 and NEAT1 strongly correlated with higher histological grades and poor prognosis. Our experiments uncovered that NEAT1 is a facultative component of the SIN3A complex, shedding light on the mechanistic actions of NEAT1 and the SIN3A complex. Further, our study identified the ER $\alpha$-NEAT1-FOXN3/ NEAT1/SIN3A-CATA3 axis that is implicated in breast cancer metastasis, providing a mechanistic insight into the pathophysiological function of FOXN3.
\end{abstract}

\section{Introduction}

The forkhead box (FOX) family of transcription factors is composed of more than 50 members that are phylogenetically classified into 19 subclasses (A to S) (1). These proteins all share a homology in their DNA-binding domain, the forkhead domain, also known as winged helix (1). FOX proteins have been implicated in a broad spectrum of cellular processes, including cell proliferation, differentiation, DNA repair, metabolism, and aging (1, 2). However, the biological functions of the mammalian forkhead transcription factors of the $\mathrm{N}$ class, including FOXN3, remain to be investigated.

It has been reported that FOXN3 is required for craniofacial and eye development in Xenopus laevis (3), and that gene inactivation of Foxn3 in mice leads to partial embryonic and postnatal lethality, growth retardation, eye formation defects, dental anomalies, and craniofacial defects (4). At the cellular level, FOXN3 was described as a checkpoint suppressor (CHES1) in yeast (5) and was shown to inhibit protein biosynthesis (6) or to downregulate E2F5 in human cells to control cell cycle (7). At the molecular level, although it has been reported that FOXN3 interacts with $x \operatorname{Sin} 3 / x R P D 3$ in $X$. laevis (3) and $\operatorname{Sin} 3$ in Saccharomyces

Conflict of interest: The authors have declared that no conflict of interest exists.

Submitted: March 31, 2017; Accepted: June 29, 2017.

Reference information: / Clin Invest. 2017;127(9):3421-3440.

https://doi.org/10.1172/JCI94233. cerevisiae (8) and with MEN1 (9) or SKIP (10) in human cells to exert transcriptional repressive function, in Drosophila testes it was demonstrated that FOXN3 acts to activate transcription (11). Clearly, the mechanistic action of FOXN3 in mammalian cells needs further elucidation. In addition, despite the observations that the expression of FOXN3 is dysregulated in tumors from a variety of tissue origins (7, 12-18), the mechanistic involvement of FOXN3 in tumorigenesis remains to be investigated, and whether and how FOXN3 plays a role in the development and progression of breast cancer are currently unknown.

Long noncoding RNAs (lncRNAs) are a class of noncoding RNAs that are over 200 nucleotides in length. The biogenesis of most lncRNAs is thought to share features with that of proteincoding mRNAs, such as splicing and polyadenylation (19-21). In recent years, lncRNAs have emerged as important components of the epigenetic regulatory network to influence transcription as well as other nuclear activities, and their dysregulation underlies several pathological states, including cancer $(20,21)$. Indeed, a number of lncRNAs have been documented to have altered expression in human cancers and have been implicated in the pathogenesis of tumors $(20,21)$. Among lncRNAs, NEAT1 (nuclear paraspeckle assembly transcript 1 ) is highly abundant and was initially identified as a structural component of nuclear paraspeckles (22). Subsequent studies suggest that NEAT1 also influences transcription either through an indirect mechanism (23) or in a direct way (24). Significantly, this lncRNA has also been implicated in malignan- 
A
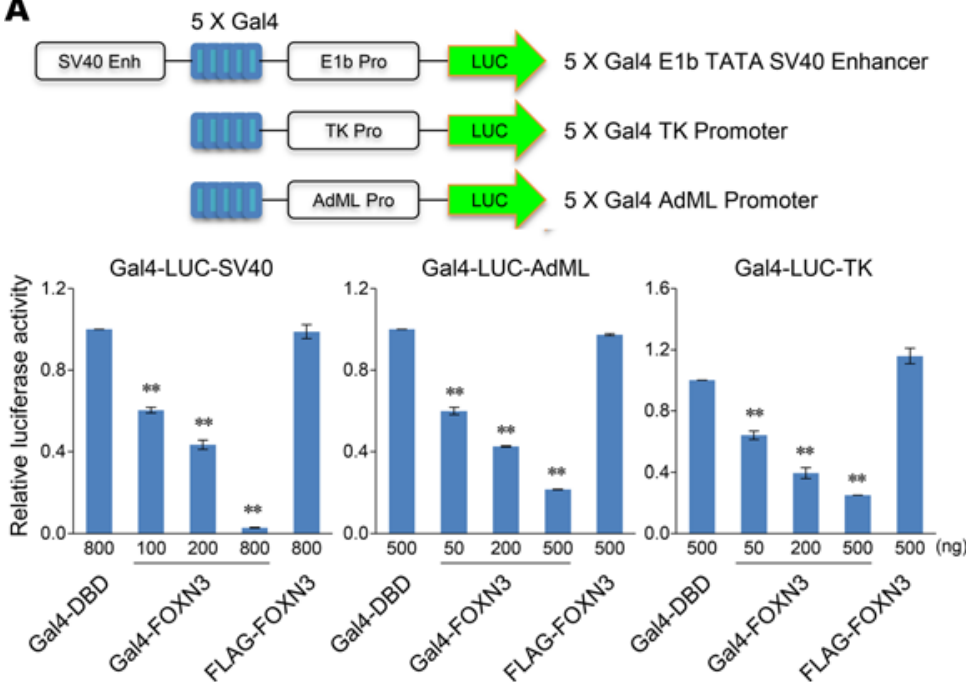

B
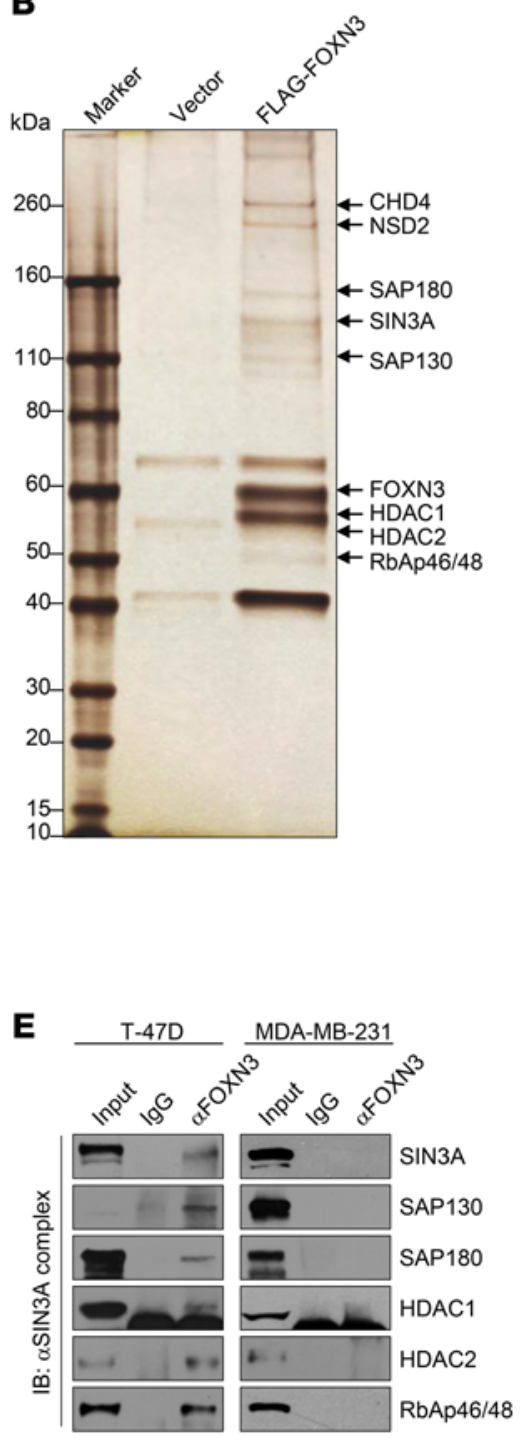

C
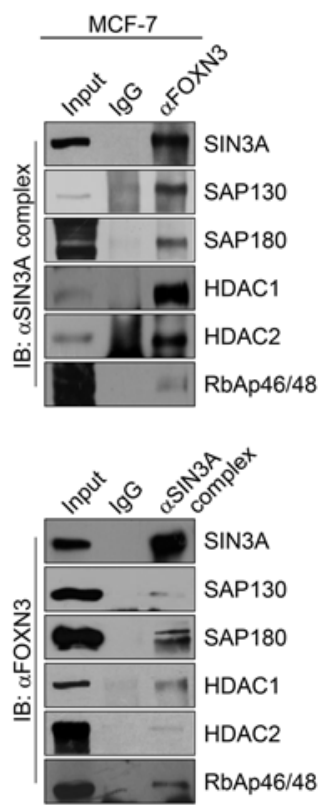

D
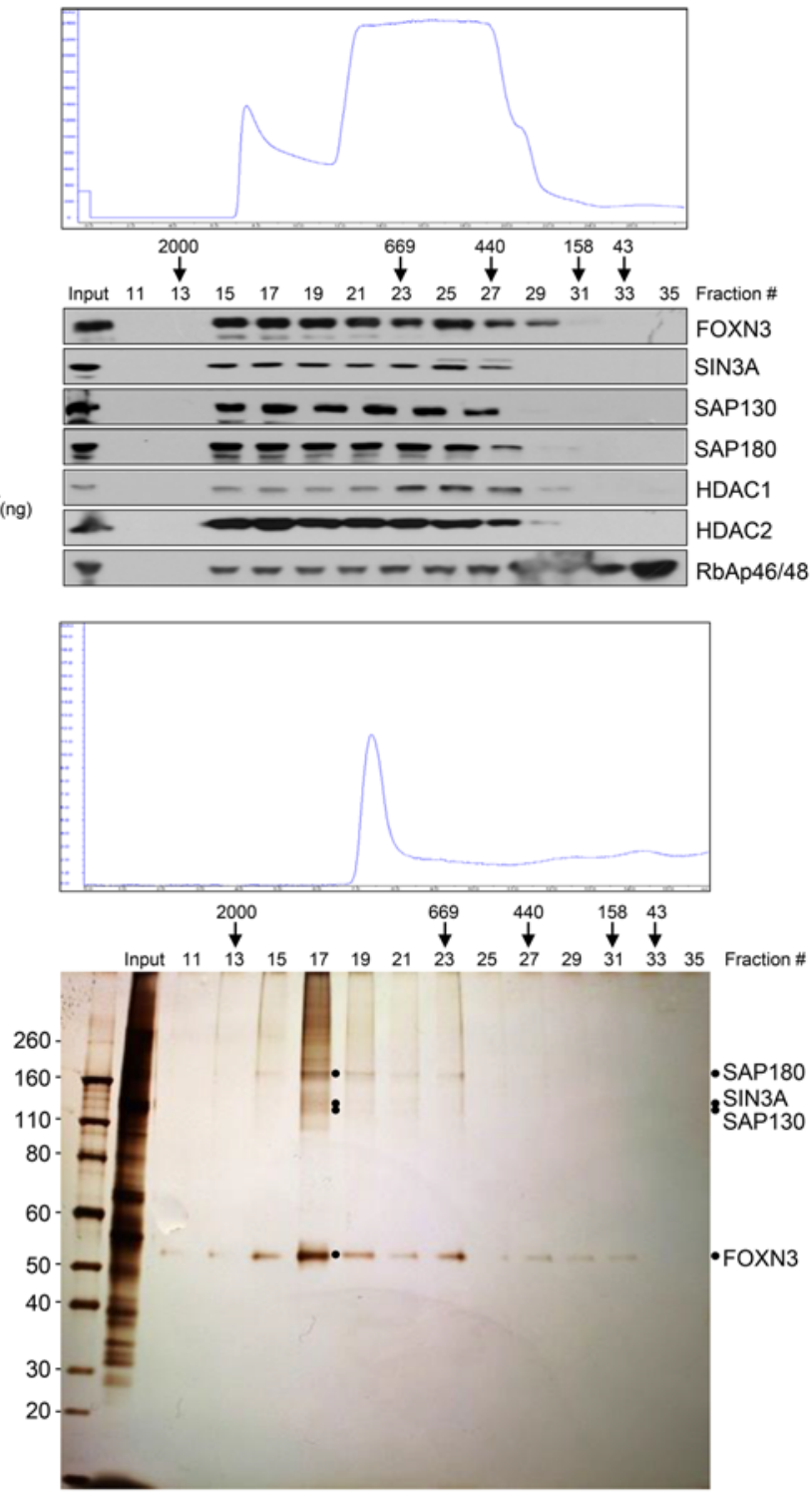

$\begin{array}{lllllllllllllll}\text { Input } & 11 & 13 & 15 & 17 & 19 & 21 & 23 & 25 & 27 & 29 & 31 & 33 & 35 & \text { Fraction \# }\end{array}$

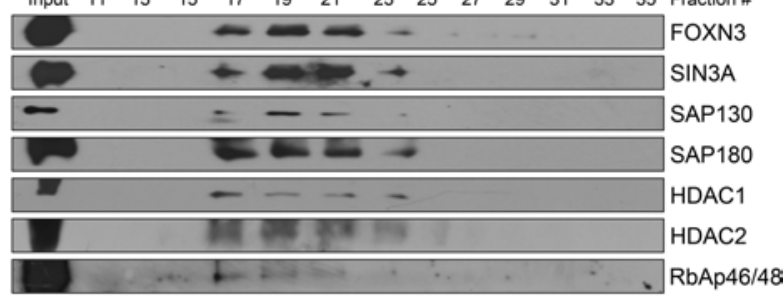

$\mathbf{F}$
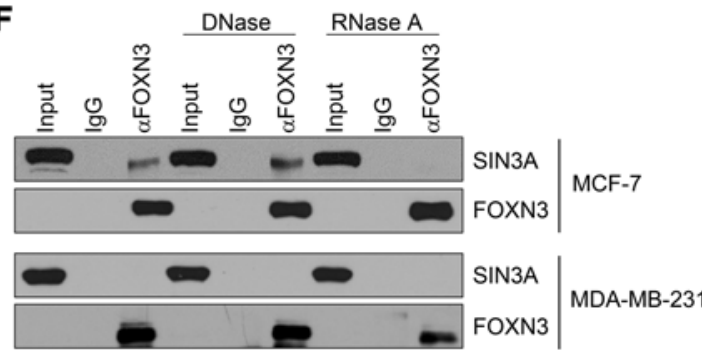
Figure 1. FOXN3 is a transcription repressor that interacts with the SIN3A complex in an RNA-dependent way. (A) Schematic diagrams of the Gal4-luciferase reporter constructs. For reporter assays, MCF-7 cells were transfected with different amounts of Gal4-FOXN3 or FLAG-FOXN3 together with the indicated Gal4-luciferase reporter. Each bar represents mean \pm SD for triplicate experiments $\left({ }^{*} P<0.01\right.$, 1-way ANOVA). (B) Immunopurification and mass spectrometry analysis of FOXN3-associated proteins. Cellular extracts from FLAG-FOXN3-expressing MCF-7 cells were affinity-purified. The eluates were resolved by SDS-PAGE and silver-stained. The protein bands were retrieved and analyzed by mass spectrometry. (C) Coimmunoprecipitation assays in MCF-7 cells with antibodies against FOXN3 followed by immunoblotting (IB) with antibodies against the indicated proteins, or with antibodies against the indicated proteins followed by IB with antibodies against FOXN3. (D) Fast protein liquid chromatography (FPLC) analysis of nuclear extracts from MCF-7 cells. Chromatographic elution profiles and IB analysis of the chromatographic fractions are shown. Equal volume from each fraction was analyzed, and the elution positions of calibration proteins with known molecular masses (kilodaltons) are indicated (top). Silver staining and Western blotting of FOXN3-containing complex fractionated by Superose 6 gel filtration (bottom). (E) Whole cell lysates from T-47D cells or MDAMB-231 cells were immunoprecipitated with antibodies against FOXN3 followed by IB with the antibodies against the indicated proteins (left). Western blotting analysis of the expression level of the indicated proteins in MCF-7, T-47D, and MDA-MB-231 cells (right). (F) Cellular lysates from MCF-7 or MDA-MB-231 cells were immunoprecipitated with antibodies against FOXN3 followed by IB with antibodies against SIN3A in the presence or absence of DNase or RNase A.

cies of a variety of tissue origins, including prostate cancer (25), ovarian cancer (26), liver cancer (27), skin cancer (28), laryngeal squamous cell cancer (29), non-small cell lung cancer (30), and glioma (31). Surprisingly, little is known about its role in breast cancer carcinogenesis, especially considering that NEAT1 is estrogen-inducible in prostate cancer cells (25).

In this study, we investigated the pathophysiological function and the underlying mechanism of FOXN3. We found that FOXN3 is physically associated with the SIN3A repressor complex and identified that NEAT1, which is induced by estrogen in breast cancer cells, is required for this interaction. We analyzed the genomic targets of the FOXN3-NEAT1-SIN3A complex and identified a panel of genes, including GATA3, that are critically involved in epithelial-to-mesenchymal transition. We found that estrogen receptor- $\alpha(\mathrm{ER} \alpha)$ itself is regulated, through negative feedback, by the FOXN3-NEAT1SIN3A complex. We investigated the role of the FOXN3-NEAT1SIN3A complex in breast cancer metastasis and explored the clinicopathological significance of the ER $\alpha$-NEAT1-FOXN3/NEAT1/ SIN3A-GATA3 axis in breast cancer progression.

\section{Results}

FOXN3 is a transcription repressor in human cells and is physically associated with the SIN3A complex in an RNA-dependent way. To further explore the biological function of FOXN3, we first investigated the transcriptional activity of this protein in human breast adenocarcinoma MCF-7 cells. For this purpose, full-length FOXN3 was fused to the C-terminus of Gal4 DNA-binding domain (Gal4FOXN3), and the transcriptional activity of the fused construct was tested in 3 different Gal4-driven luciferase reporter systems, which all contain 5 copies of Gal4 binding sequence but differ in basal promoter element (Figure 1A). The results showed that Gal4FOXN3 exhibited a robust repression of the reporter activity in a dose-dependent fashion in all of the 3 reporter systems (Figure 1A). However, overexpression of FLAG-tagged FOXN3 (FLAGFOXN3) did not affect the activity of the Gal4-driven reporters, suggesting that FOXN3 must be physically associated with DNA to exert its repression activity (Figure 1A).

In order to gain mechanistic insights into the transcription repression function of FOXN3, we used affinity purification and mass spectrometry to interrogate FOXN3 interactome in vivo. In these experiments, FLAG-FOXN3 was stably expressed in MCF-7 cells. Cellular extracts were prepared and subjected to affinity purification using an anti-FLAG affinity column. After extensive washing, the bound proteins were eluted with excess FLAG peptides, resolved, and visualized by silver staining on SDS-PAGE. The protein bands on the gel were recovered and analyzed by mass spectrometry. The results revealed that FOXN3 was copurified with SIN3A, SAP130, SAP180, HDAC1, HDAC2, RbAp46, and RbAp48, all components of the SIN3A complex, as well as several other proteins (Figure 1B). The detailed result of the mass spectrometric analysis is provided in Supplemental Table 1 (supplemental material available online with this article; https://doi.org/10.1172/JCI94233DS1).

To confirm the in vivo interaction between FOXN3 and the SIN3A complex, total proteins from MCF-7 cells were extracted, and coimmunoprecipitation was performed with antibodies detecting the endogenous proteins. Immunoprecipitation (IP) with antibodies against FOXN3 followed by immunoblotting (IB) with antibodies against SIN3A, SAP130, SAP180, HDAC1, HDAC2, or RbAp46/48 demonstrated that all these proteins were efficiently coimmunoprecipitated with FOXN3 (Figure 1C). Reciprocally, IP with antibodies against representative components of the SIN3A complex and IB with antibodies against FOXN3 also showed that FOXN3 was efficiently coimmunoprecipitated with the components of the SIN3A complex (Figure 1C).

To further support the physical association of FOXN3 with the SIN3A complex, MCF-7 nuclear proteins were fractionated by fast protein liquid chromatography (FPLC) on Superose 6 columns with a high-salt extraction and size exclusion approach. Notably, native FOXN3 from MCF-7 cells was eluted with an apparent molecular mass much greater than that of the monomeric protein; FOXN3 was detected in chromatographic fractions from Superose 6 column with a peak centered between approximately 669 and 2,000 kDa. Significantly, the elution pattern of FOXN3 largely overlapped with that of the SIN3A components (Figure 1D, top). In addition, analysis of FLAG-FOXN3 affinity elutes from FPLC after Superose 6 gel filtration revealed that the majority of the purified FLAG-FOXN3 existed in a multiprotein complex, which peaked in fractions 17 and 19 and contained subunits of the SIN3A complex (Figure 1D, bottom).

To further consolidate the observation that FOXN3 is physically associated with the SIN3A complex, coimmunoprecipitation experiments were performed in additional cell lines, including breast cancer cell lines T-47D (ER $)$ and MDA-MB-231 (ER-). IP with antibodies against FOXN3 followed by IB with antibodies against representative components of the SIN3A complex showed that the physical interaction between FOXN3 and the SIN3A complex was also detected in T-47D, but not in MDAMB-231 cells (Figure 1E, left). The lack of detectable interaction between FOXN3 and the SIN3A complex in MDA-MB-231 cells 
A

FOXN3 IRIP-Seq

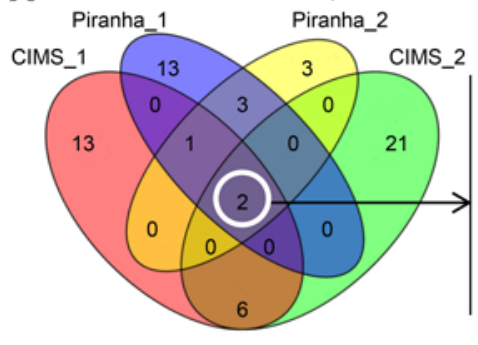

B

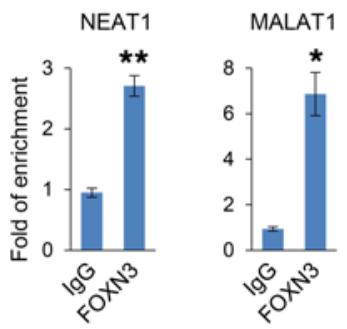

E

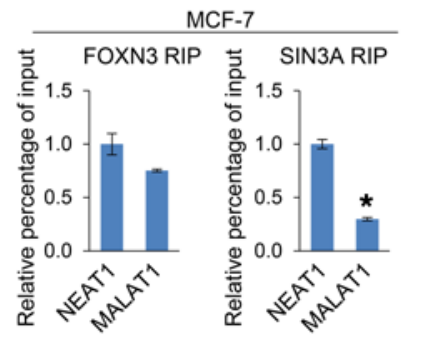

F
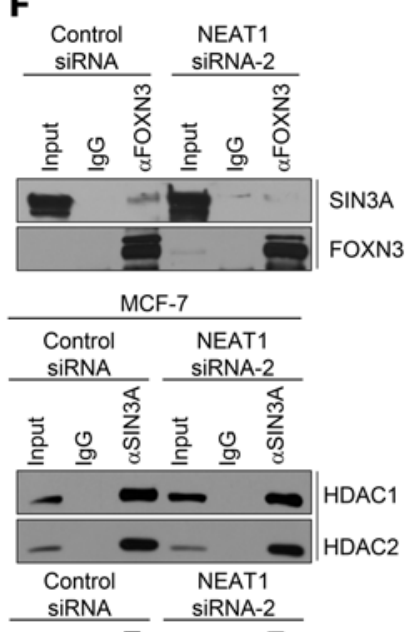

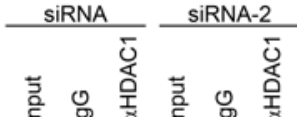

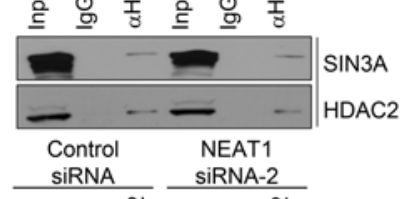

$\frac{\text { SiRNA }}{\text { ปู }} \frac{\text { SIRNA-2 }}{\text { ปั }}$
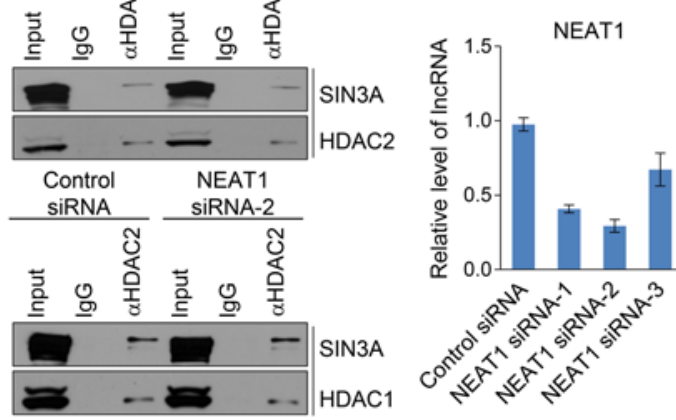
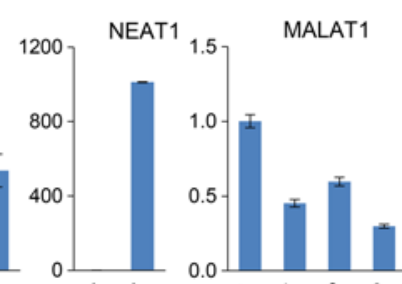

RCSs| I | ||

704-903 nt (in vitro-transcribed fragment)

RCSS| III I I III I| I I III I I
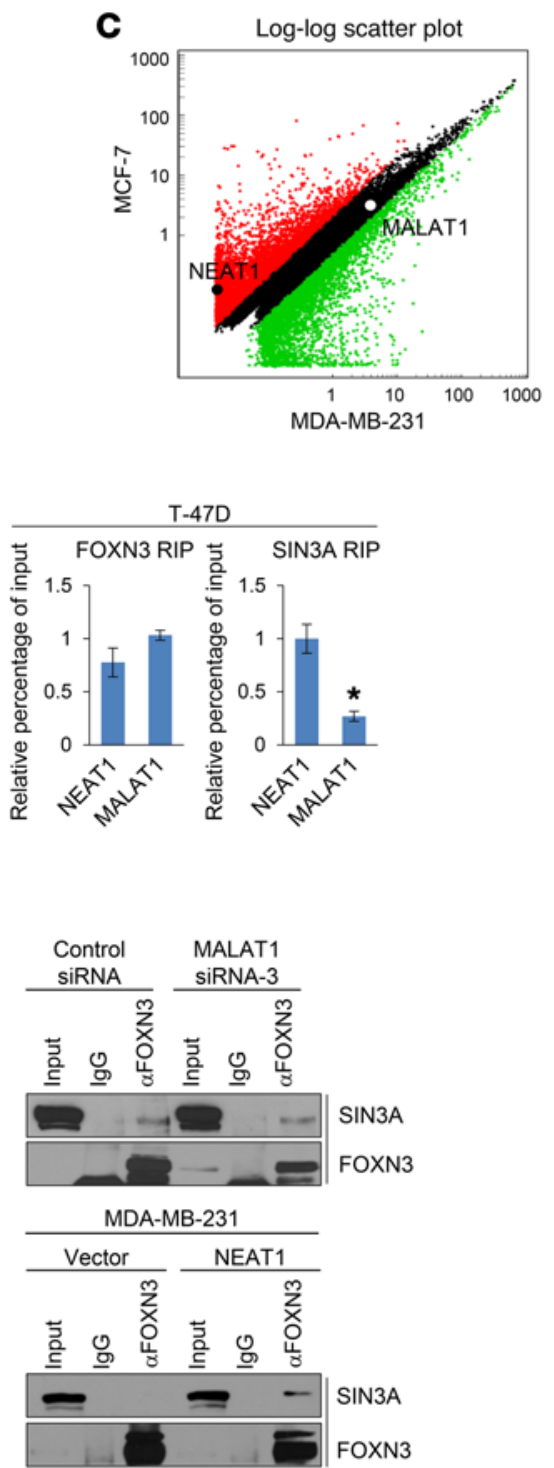

$3 x^{2}+x^{2} x^{3}$

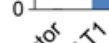

0.0

$5^{2}+x^{2} x^{3}$

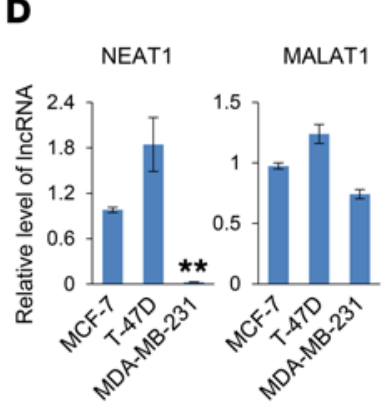

G
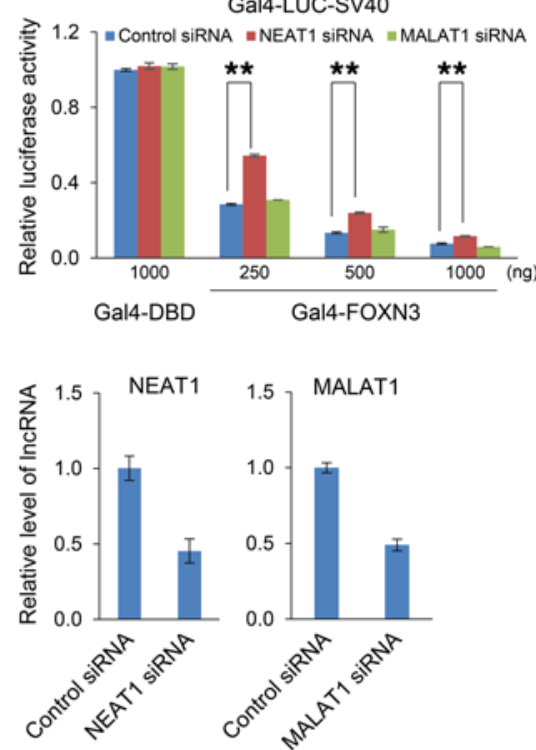

H

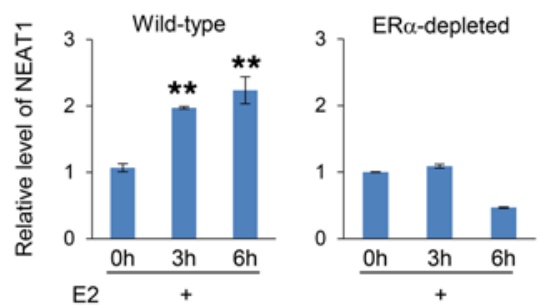


Figure 2. NEAT1 is required for the interaction of FOXN3 with the SIN3A complex. (A) Venn diagrams of FOXN3 iRIP-Seq results for potential IncRNAs (left). The RNA contact sites (RCSs) that represent the most frequent tags are mapped to the sequences of NEAT1 and MALAT1 (right). NEAT1 fragment used for in vitro RNA pull-down is indicated. (B) RIP-qPCR verification of the IRIP-Seq results with antibodies against the indicated proteins in MCF-7 cells. Error bars represent mean \pm SD for triplicate experiments ( ${ }^{*} P<0.05,{ }^{* *} P<0.01 ; t$ test). (C) Scatter plot of microarray profiling of IncRNA expression in the indicated cell lines. (D) qPCR analysis of the expression of NEAT1 and MALAT1 in the indicated cell lines. Error bars represent mean $\pm S D$ for triplicate experiments ${ }^{* *} P<0.01$, 1-way ANOVA). (E) RIP-qPCR analysis of FOXN3 RIP or SIN3A RIP for the enrichment of NEAT1 and MALAT1 in MCF-7 and T-47D cells. Error bars represent mean \pm SD for triplicate experiments $\left({ }^{*} P<0.05, t\right.$ test). (F) IP in MCF-7 cells depleted with NEAT1 or MALAT1 using anti-FOXN3 followed by IB with anti-SIN3A (top) or IP with anti-HDAC1, anti-HDAC2, or anti-SIN3A followed by IB with the indicated antibodies (bottom left). Cellular lysates from NEAT1-overexpressed MDA-MB-231 cells were immunoprecipitated with anti-FOXN3 followed by IB with anti-SIN3A (bottom right). Knockdown efficiency was verified by qPCR. (C) Reporter assays in NEAT1- or MALAT1-depleted MCF-7 cells. Knockdown efficiency was verified by qPCR. Error bars represent mean \pm SD for triplicate experiments $\left({ }^{* *} P<0.01\right.$, 2-way ANOVA). (H) Wild-type or ER $\alpha$-depleted MCF-7 cells were deprived of steroids before treatment with E2 for the indicated times. Total RNAs were extracted for qPCR analysis of the expression of NEAT1. Error bars represent mean $\pm S D$ for triplicate experiments ( ${ }^{* *} P<0.01$, 1-way ANOVA).

was not due to the absence of FOXN3 or the SIN3A complex, as Western blotting analysis showed FOXN3, SIN3A, SAP130, SAP180, HDAC1, HDAC2, and RbAp46/48 in all of these cell lines, with the level of FOXN3 even higher in MDA-MB-231 cells than in T-47D cells (Figure 1E, right).

It is puzzling that the interaction between FOXN3 and the SIN3A complex, while detected in MCF-7 and T-47D cells, is absent in MDA-MB-231 cells, yet the proteins involved are nevertheless expressed in these cells. A simple explanation is that an additional element(s)/component(s) beyond FOXN3 and the SIN3A complex is required for their interaction, which is present in MCF-7 and T-47D cells and absent in MDA-MB-231 cells. In this regard, we were intrigued by the findings implicating lncRNAs in protein-protein interaction (32-34) and thus hypothesized that the interaction between FOXN3 and the SIN3A complex might also involve an lncRNA. To test this, whole cell lysates were prepared from MCF-7 and MDA-MB-231 cells, and coimmunoprecipitation experiments were performed in the presence or absence of DNase or RNase A. IP with antibodies against FOXN3 followed by IB with antibodies against SIN3A detected the interaction of FOXN3 with SIN3A in cellular lysates from MCF-7 cells, but only in the absence of RNase A; in the presence of RNase A, the interaction between FOXN3 and SIN3A was no longer detected (Figure 1F). No interaction between FOXN3 and SIN3A was detected in cellular lysates from MDA-MB-231 cells, regardless of the presence or absence of RNase A (Figure 1F). Together, these results support a notion that the interaction between FOXN3 and the SIN3A complex requires an RNA molecule.

LncRNA NEAT1 is required for the interaction of FOXN3 with the SIN3A complex. To identify the RNA molecule that is required for the interaction of FOXN3 with the SIN3A complex, we performed improved RNA immunoprecipitation-coupled high-throughput sequencing (iRIP-Seq) in MCF-7 cells in which RNAs/proteins were UV-cross-linked in vivo and RNA-bound proteins were immunoprecipitated with antibodies against FOXN3 followed by MNase treatment and RNA extraction for paired-end deep sequencing using the Illumina NextSeq 500 system. The experiments were biologically repeated twice (replicate 1 and replicate 2). The raw reads were mapped to the human reference genome (GRCh38/hg38) via TopHat, a fast splice junction mapper for RNASeq reads, with default parameters. After subtraction of duplicate alignments, replicate 1 yielded a total of 3,246,724 tags for FOXN3 iRIP and 331,880 tags for IgG iRIP, and replicate 2 produced a total of 3,650,068 tags for FOXN3 iRIP and 607,260 tags for IgG iRIP. These tags were then analyzed for FOXN3-associated RNA sequences by 2 computational methods: (a) CIMS (cross-linkinginduced mutation site), which is based on the reverse transcription error frequency in cDNAs induced by UV cross-linking, and (b) Piranha (http://smithlab.usc.edu), which is based on zero-truncated negative binomial. Intercrossing the sequence tags from the 2 replicates by the 2 different computations followed by screening for noncoding exons identified 2 lncRNAs, NEAT1 and MALAT1, as potential FOXN3-associated RNA molecules (Figure 2A). The detailed results from the iRIP-Seq experiments are deposited in the NCBI's Gene Expression Omnibus (GEO GSE93781).

RNA immunoprecipitation-coupled real-time quantitative reverse transcription PCR (qPCR) was then performed in MCF-7 cells in which UV cross-linking was followed by IP with antibodies against FOXN3. Subsequent qPCR analysis showed enrichment of NEAT1 and MALAT1, validating the iRIP-Seq results (Figure 2B). To determine which lncRNA, NEAT1 or MALAT1, participates in the interaction between FOXN3 and SIN3A, we first performed a comparative analysis of the expression profiles of lncRNAs in MCF-7 and MDA-MB-231 cells using lncRNA microarrays. The results showed that the level of NEAT1 expression was massively higher in MCF-7 cells than in MDA-MB-231 cells, whereas the levels of MALAT1 were comparable in these 2 cell lines (Figure 2C). Normalized fold of changes of NEAT1 or MALAT1 in MCF-7 cells over MDA-MB-231 cells is shown in Supplemental Table 2. The detailed results from the microarray experiments are deposited in GEO (GSE93781). Analysis by qPCR for the expression of NEAT1 and MALAT1 in MCF-7, T-47D, and MDA-MB-231 cells verified the results (Figure 2D).

RNA immunoprecipitation-coupled qPCR (RIP-qPCR) was then performed in MCF-7 and T-47D cells with antibodies against FOXN3 or SIN3A. Subsequent qPCR analysis showed that the enrichment of NEAT1 was significantly higher than that of MALAT1 in SIN3A RNA immunoprecipitation (RIP), while the enrichments of NEAT1 and MALAT1 in FOXN3 RIP were comparable (Figure 2E). Moreover, coimmunoprecipitation experiments in MCF-7 cells showed that the interaction between FOXN3 and the SIN3A complex was largely abrogated upon knockdown of NEAT1, but not MALAT1 (Figure 2F, top), although the integrity of the SIN3A complex was not affected by NEAT1 depletion (Figure 2F, bottom left). Meanwhile, the interaction between FOXN3 and the SIN3A complex became detectable in MDA-MB-231 cells when NEAT1 was forcibly expressed (Figure $2 \mathrm{~F}$, bottom right). Moreover, reporter assays with Gal4-driven luciferase in MCF-7 cells demonstrated that knockdown of NEAT1, but not MALAT1, significantly compromised the transcription repression of the reporter activity by 
A
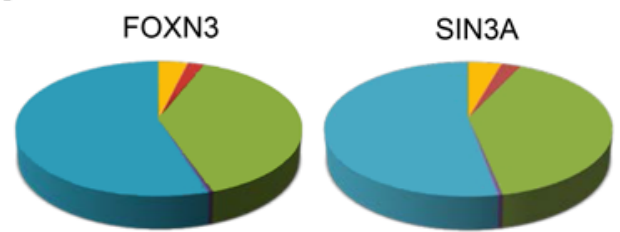

= Promoter

Exon

= Intron

- Downstream

= Distal Intergenic

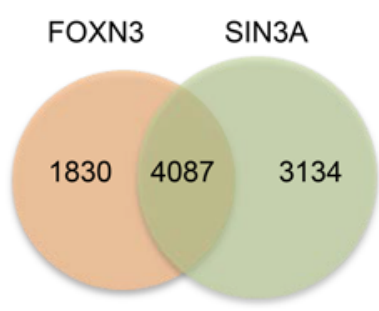

B

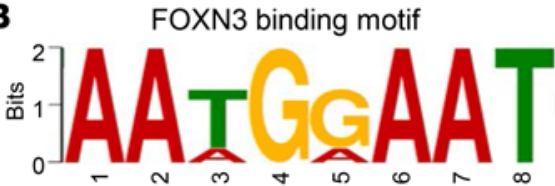

SIN3A binding motif
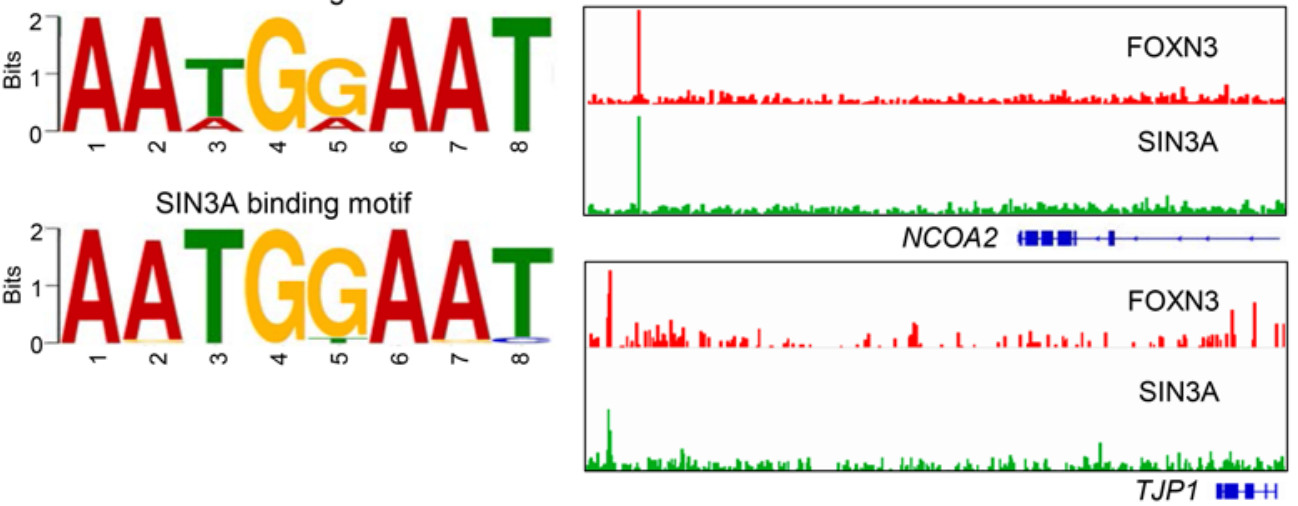

C
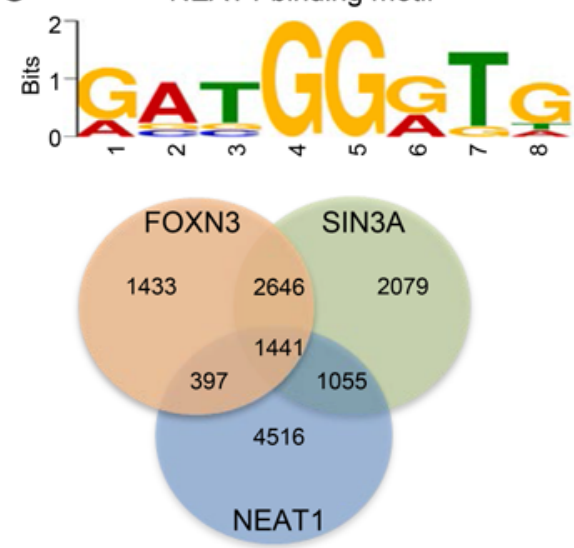

D

" Control siRNA $=$ FOXN3 siRNA

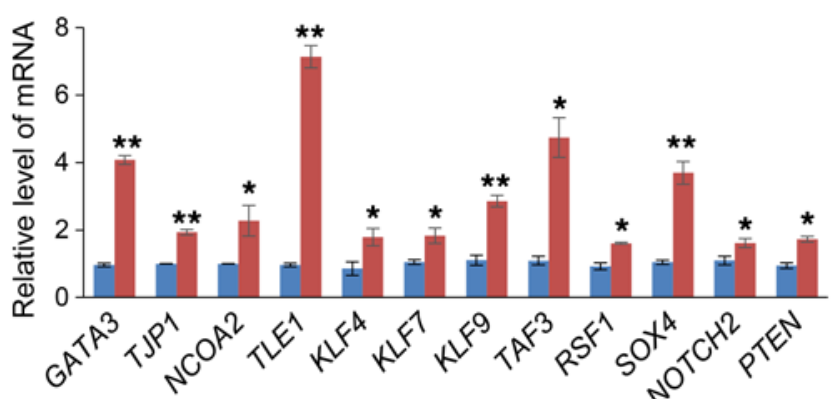

FOXN3 (Figure 2G). Collectively, these results support a notion that lncRNA NEAT1 is required for the formation and function of the FOXN3-SIN3A complex.

As stated earlier, NEAT1 was reported to be an estrogeninducible lncRNA in prostate cancer cells (25). In light of our observation that the interaction of FOXN3 with the SIN3A com-
E

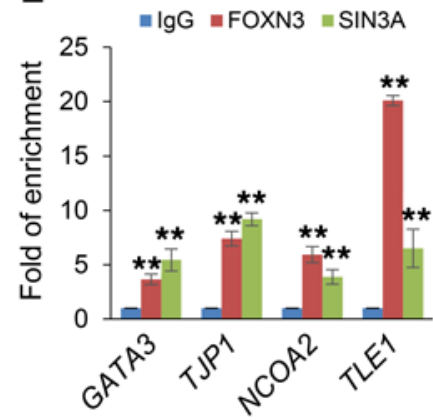

Figure 3. Genome-wide analysis of the transcriptional targets of the FOXN3-NEAT1-SIN3A complex.

(A) Genomic distribution of the transcriptional targets of FOXN3 and SIN3A based on ChIP-Seq data (left). Venn diagrams of overlapping genes targeted by FOXN3 and SIN3A in MCF-7 cells (right). (B) MEME-ChIP analysis of the DNA binding motifs of FOXN3 and SIN3A (left). The binding profiles of FOXN3 and SIN3A on NCOA2 and TJP1 are shown (right). (C) MEMEChIP analysis of the DNA binding motifs of NEAT1. Venn diagrams of overlapping genes targeted by FOXN3, SIN3A, and NEAT1 in MCF-7 cells based on FOXN3-SIN3A ChIPSeq data and NEAT1 CHART-Seq data (left). Density distributions (read count per million of mapped reads) of SIN3A, NEAT1, and MALAT1 peaks on the transcription start site (TSS) of FOXN3-enriched genes were analyzed by ngs.plot (right). (D) qPCR measurement of the expression of the indicated genes in MCF-7 cells under FOXN3 depletion. Error bars represent mean \pm SD for triplicate experiments $\left({ }^{*} P<0.05,{ }^{* *} P<0.01 ; t\right.$ test). (E) qChIP verification of the ChIP-Seq results on the promoter of the indicated genes with antibodies against the indicated proteins in MCF-7 cells. Error bars represent mean \pm SD for triplicate experiments ( ${ }^{* *} P<0.01,2$-way ANOVA). plex was detected in ER $\mathrm{E}^{+} \mathrm{MCF}-7$ and T-47D cells but not in $\mathrm{ER}^{-}$ MDA-MB-231 cells, it is logical to postulate that NEAT1 is induced by estrogen in breast cancer cells. To test this, MCF-7 cells were deprived of steroids for 3 days prior to treatment with $17 \beta$-estradiol (E2) for different times. Total RNAs were extracted, and the expression of NEAT1 was analyzed by qPCR. The results showed 

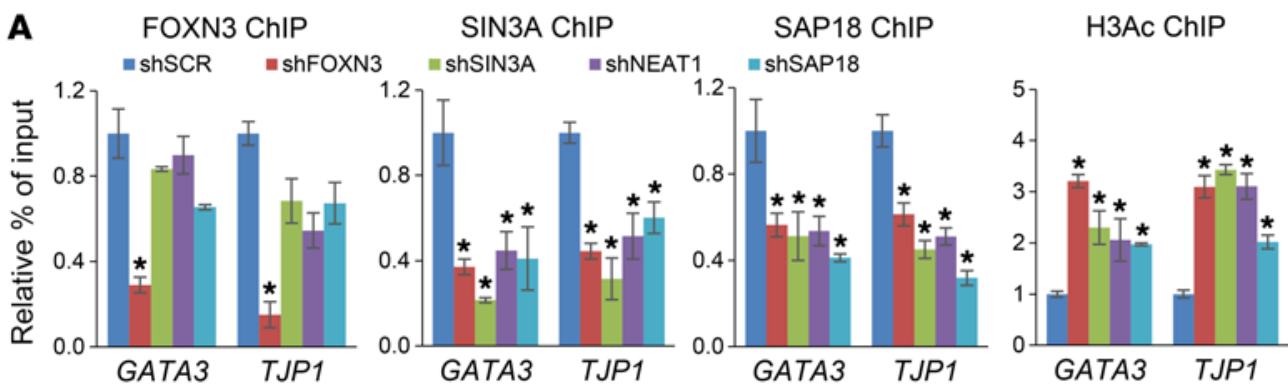

B

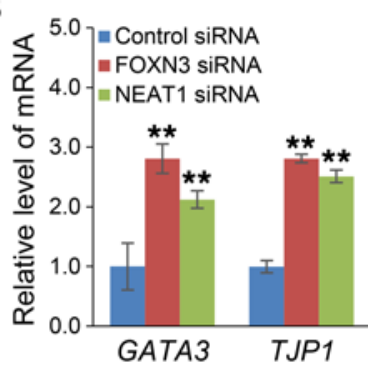

H3 ChIP
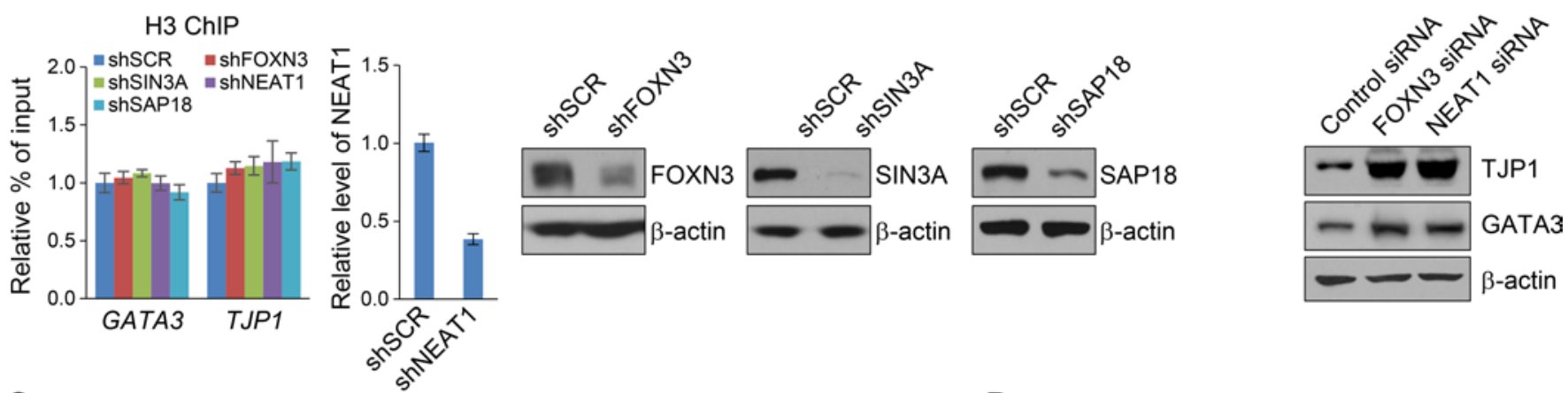

C
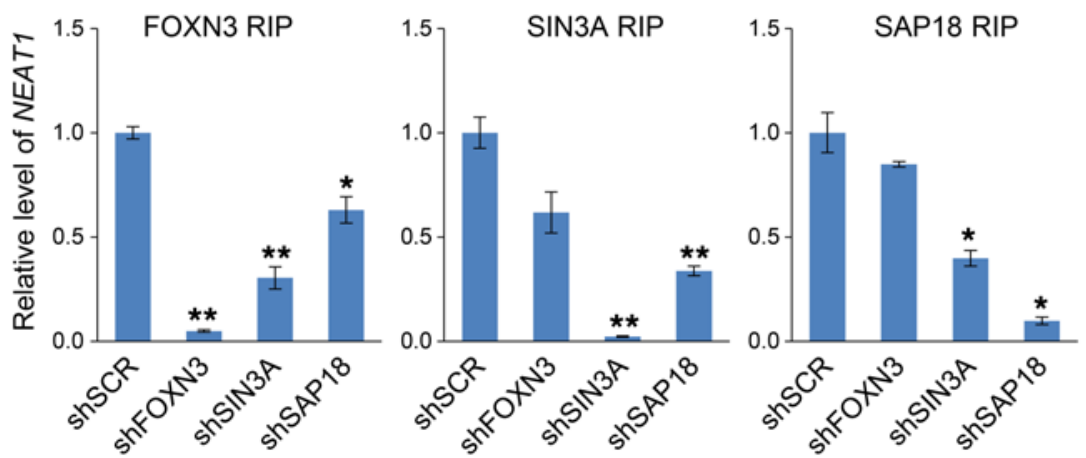

D

\section{Predicted RBRs}
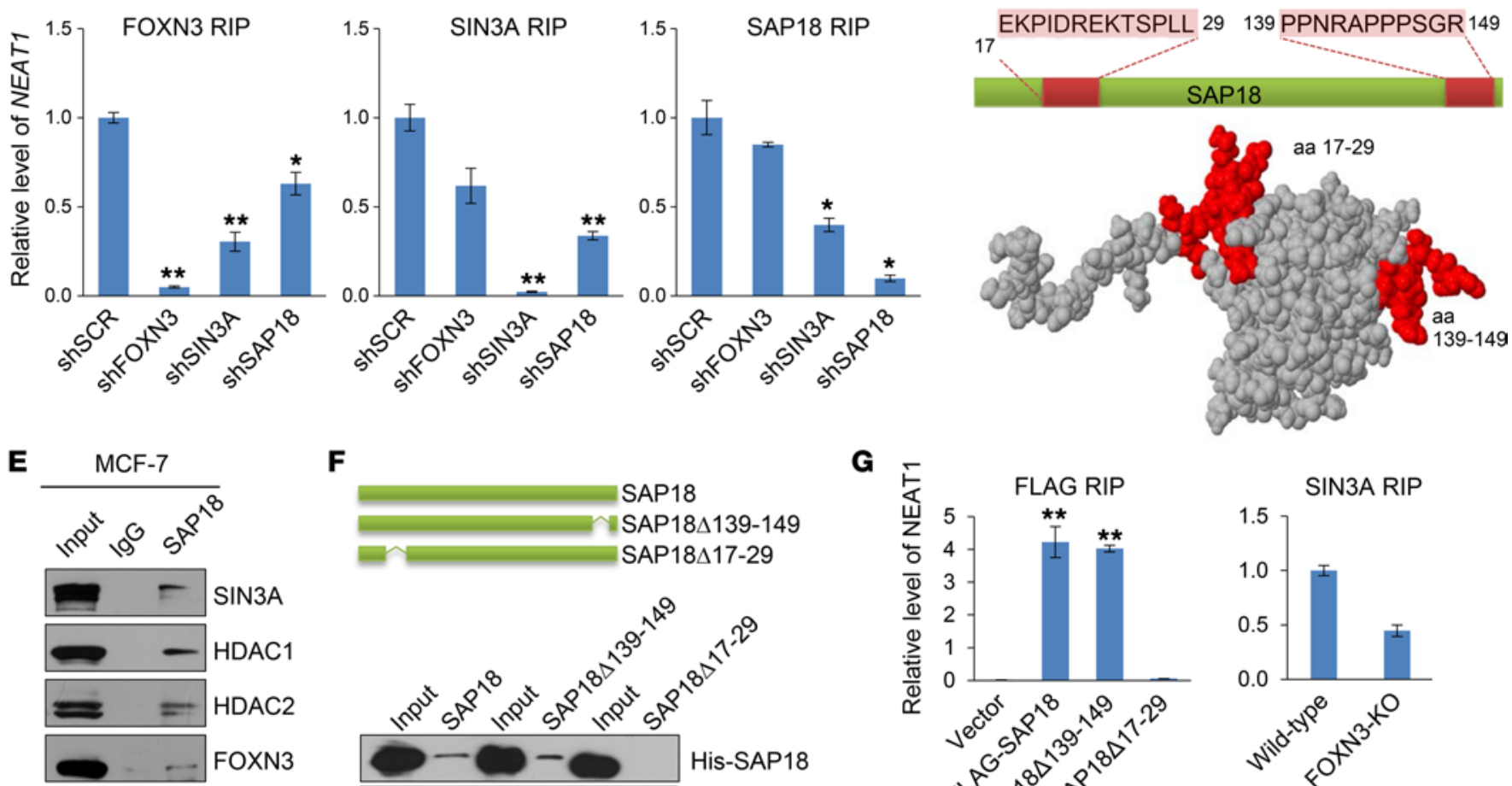

$\mathbf{F}$
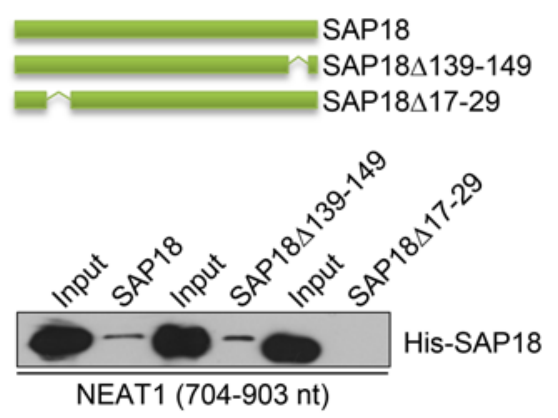

G

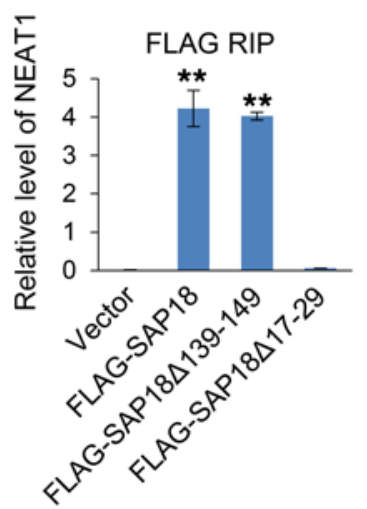

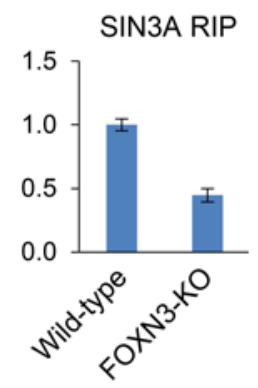

Figure 4. The assembly of the FOXN3-NEAT1-SIN3A complex on transcriptional targets. (A) MCF-7 cells were infected with lentiviruses carrying the indicated shRNAs for qChIP analysis on the selected promoters using antibodies against the indicated proteins/histone modification. Error bars represent mean \pm SD for triplicate experiments. Knockdown efficiency was verified by qPCR or Western blotting. (B) qPCR and Western blotting analyses of the expression of CATA3 and TJP1 (ZO1) in MCF-7 cells transfected with the indicated siRNAs. In A and $\mathbf{B}$, error bars represent mean \pm SD for triplicate experiments ( ${ }^{*} P<0.05,{ }^{* *} P<0.01$; 2-way ANOVA). (C) RIP-qPCR analysis of FOXN3 RIP, SIN3A RIP, or SAP18 RIP for the enrichment of NEAT1 in MCF-7 cells infected with lentiviruses carrying the indicated shRNAs. (D) Prediction of RNA-binding residues (RBRs) in SAP18 by RBRDetector. The predicted RBRs (red) in the 3D structure of SAP18 are visualized by Jmol (www.jmol.org/). (E) Coimmunoprecipitation assays in MCF-7 cells with antibodies against SAP18 followed by IB with antibodies against the indicated proteins. (F) Schematic diagrams of GST/His-SAP18, GST/His-SAP18 $17-29$, and GST/His-SAP18 139 -149. The results of RNA pull-down assays with NEAT1 fragment (704-903 nt) and GST/His-SAP18, GST/His-SAP18 $17-29$, or GST/ His-SAP18 139 -149 are shown. (C) RIP-qPCR analysis of FLAG RIP for the enrichment of NEAT1 in MCF-7 cells transfected with FLAG-SAP18, FLAGSAP18 17 -29, or FLAG-SAP18 139-149 (left). RIP-qPCR analysis of SIN3A RIP for the enrichment of NEAT1 in a FOXN3-knockout MCF-7 cell line established by the CRISPR/Cas9 system (right). In C and G, error bars represent mean \pm SD for triplicate experiments ( ${ }^{*} P<0.05$, ${ }^{* *} P<0.01 ; 1$-way ANOVA). 
that NEAT1 is clearly induced by estrogen in MCF-7 cells (Figure $2 \mathrm{H})$. Consistently, when ER $\alpha$ was knocked down in MCF-7 cells, estrogen no longer induced the expression of NEAT1 (Figure 2H).

Identification of genome-wide transcriptional targets for the FOXN3-NEAT1-SIN3A complex. In order to explore the functional significance of the physical association between FOXN3 and the SIN3A complex, we next analyzed the genome-wide transcriptional targets of FOXN3 and SIN3A by chromatin immunoprecipitation-based deep sequencing (ChIP-Seq) in MCF-7 cells. Following ChIP, FOXN3- and SIN3A-associated DNAs were amplified using nonbiased conditions, labeled, and sequenced using BGISEQ-500 (BGI). We identified 22,816 FOXN3-specific binding peaks and 31,986 SIN3A-specific binding peaks. The detailed results from the ChIP-Seq experiments are deposited in GEO (GSE93781). The ChIP-Seq peak data are analyzed in Figure 3A.

The data from FOXN3 ChIP-Seq and SIN3A ChIP-Seq were then cross-analyzed for overlapped DNA sequences, and these sequences were considered to be the targets of the FOXN3-SIN3A complex. These experiments identified a total of 4,087 genes targeted by the FOXN3-SIN3A complex (Figure 3A, right). Significantly, FOXN3 and SIN3A had very similar binding motifs, and FOXN3 and SIN3A exhibited similar peak locations on the representative target genes (Figure 3B), supporting the physical interaction and functional connection between FOXN3 and SIN3A.

The FOXN3-SIN3A ChIP-Seq results were then cross-analyzed with the published CHART-Seq data for NEAT1 in MCF-7 cells (24) for overlapped sequences, and these sequences were considered to be the genomic targets of the FOXN3-NEAT1-SIN3A complex (Figure 3C). CHART-Seq (capture hybridization analysis of RNA targets-based deep sequencing) is a technique using biotinylated antisense oligonucleotides to capture RNA from cross-linked chromatin extracts (35). These analyses identified a total of 1,441 genes that are targeted by the FOXN3-NEAT1-SIN3A complex, including GATA3 and TJP1, which are well recognized for their roles in the regulation of mammary epithelium differentiation under normal physiology and epithelial-to-mesenchymal transition (EMT) and cell invasion during breast cancer progression (36-38). Significantly, NEAT1 also had similar genomic signatures to that of FOXN3 and SIN3A, and, remarkably, analysis by ngs.plot, a Java-based tool for quick mining and visualization of high-throughput sequencing data (39), showed similar genomic landscapes for both SIN3A and NEAT1 on FOXN3-targeted sites, whereas MALAT1 lacked such a characteristic (Figure 3C). In addition, qPCR measurement of a panel of target genes in MCF-7 cells showed that depletion of FOXN3 resulted in a significant increase, albeit to different extents, in the expression of these genes (Figure 3D). Moreover, quantitative ChIP (qChIP) analysis in MCF-7 cells using specific antibodies against FOXN3 or SIN3A on selected genes including GATA3, TJP1, NCOA2, and TLE1 showed strong enrichment of FOXN3 and SIN3A on the promoters of these genes (Figure 3E). Together, these findings support the physical interaction and functional connection among FOXN3, NEAT1, and SIN3A.

The assembly of the FOXN3-NEAT1-SIN3A complex on transcriptional targets. To further support that FOXN3 and the SIN3A complex occupy the target genes in the context of the FOXN3SIN3A complex, sequential ChIP or ChIP/Re-ChIP experiments were performed on representative target genes including GATA3,
$T J P 1, N C O A 2$, and TLE1. In these experiments, soluble chromatins were first immunoprecipitated with antibodies against FOXN3 or SIN3A. The immunoprecipitates were subsequently reimmunoprecipitated with appropriate antibodies. The results showed that, in precipitates, the GATA3, TJP1, NCOA2, and TLE1 promoters that were immunoprecipitated with antibodies against FOXN3 could be reimmunoprecipitated with antibodies against SIN3A (Supplemental Figure 1). Similar results were obtained when initial ChIP was done with antibodies against SIN3A (Supplemental Figure 1). These results support a notion that FOXN3 and SIN3A bind the target genes as 1 protein complex.

To investigate the functional significance of NEAT1 in the formation and chromatin targeting of the FOXN3-NEAT1-SIN3A complex, MCF-7 cell clones with FOXN3, SIN3A, SAP18, or NEAT1 stably depleted were generated by lentivirus-delivered shRNA. qChIP experiments in these cells showed that depletion of FOXN3 resulted in a diminished recruitment of FOXN3 as well as SIN3A and SAP18 on the promoters of GATA3 and TJP1 (Figure 4A). Interestingly, however, while depletion of either SIN3A, SAP18, or NEAT1 led to a diminished recruitment of SIN3A and SAP18 on the promoters of GATA3 and TJP1, it had only marginal effect on the recruitment of FOXN3 on these promoters (Figure 4A). Consistently, qChIP assays showed that depletion of FOXN3, SIN3A, or NEAT1 in MCF-7 cells was associated with a significant increase in the level of $\mathrm{H} 3$ acetylation (H3Ac) on the promoters of GATA3 and TJP1 (Figure 4A), and qPCR and Western blotting experiments demonstrated that depletion of either FOXN3 or NEAT1 in MCF-7 cells led to an increased expression of GATA3 and TJP1 (Figure 4B). Collectively, these results point to a scenario in which FOXN3 acts to recruit or enlist NEAT1 and the SIN3A complex to the genomic targets to repress the transcription of these targets.

The observation that depletion of NEAT1 affected the genomic binding of SIN3A and SAP18 but not that of FOXN3 is intriguing. To further understand the genomic targeting of the FOXN3NEAT1-SIN3A complex, FOXN3, SIN3A, or SAP18 was individually knocked down in MCF-7 cells, and RIP-qPCR experiments were performed in these cells using antibodies against FOXN3, SIN3A, or SAP18 to detect the enrichment of NEAT1. We found that while depletion of FOXN3 had marginal effects on the enrichment of NEAT1 in SIN3A RIP and SAP18 RIP, depletion of either SIN3A or SAP18 resulted in a significant decrease in the enrichment of NEAT1 in FOXN3 RIP (Figure 4C). Notably, depletion of SAP18 led to a decrease in the enrichment of NEAT1 in SIN3A RIP (Figure 4C, middle). These results point to a stoichiometry of the formation of the FOXN3-NEAT1-SIN3A complex in which NEAT1 is closely associated with the SIN3A complex, suggesting that NEAT1 is a facultative component of the SIN3A complex.

It is believed that RNA-protein interaction involves RNAbinding residues (RBRs) in the protein (40). To support the proposition that NEAT1 is a facultative component of the SIN3A complex, we first analyzed the components of the SIN3A complex as well as FOXN3 for RBRs and found that only SAP18 contains potential RBRs (Figure 4D). The detailed result of the prediction of RBRs in SAP18 by RBRDetector (http://ibi.hzau.edu.cn/ rbrdetector) is provided in Supplemental Table 3. Indeed, a previous quantitative proteomics screening for RNA-binding proteins identified SAP18 as an RNA-binding protein (41). To confirm that 
A

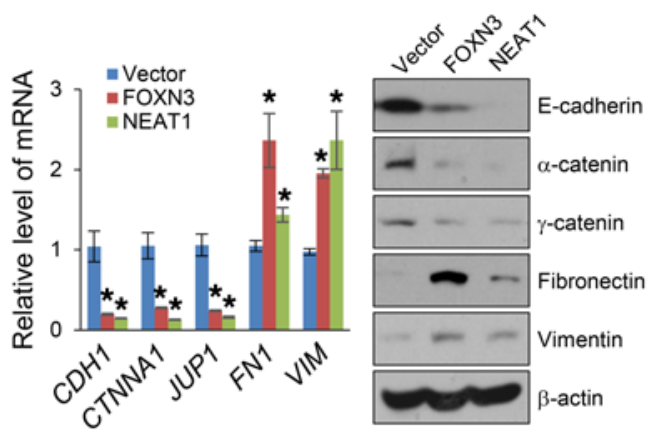

B

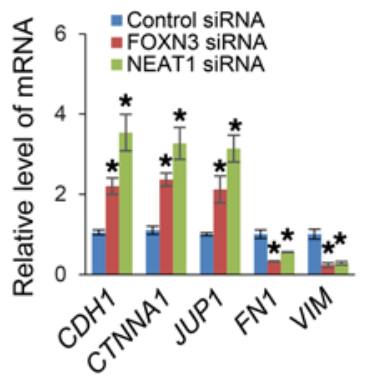

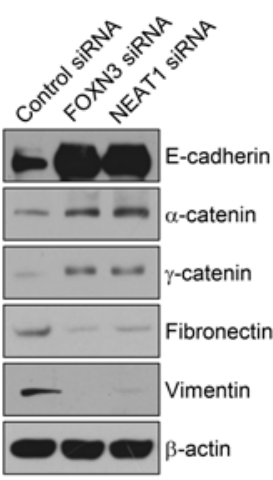

C

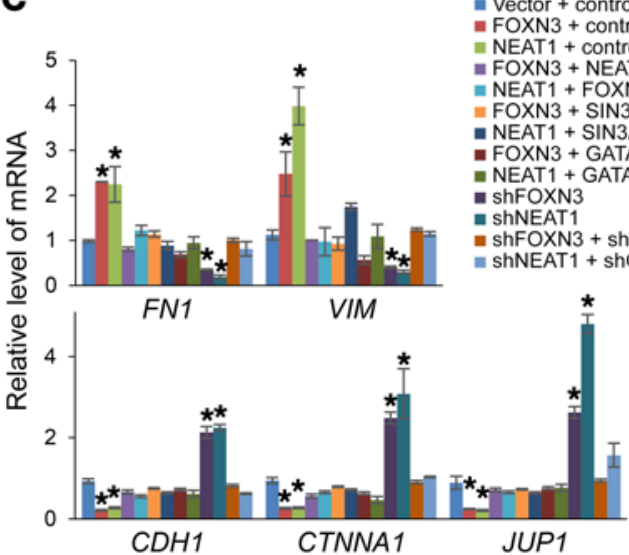

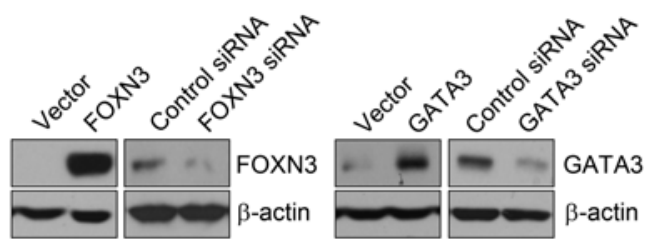

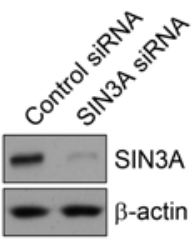

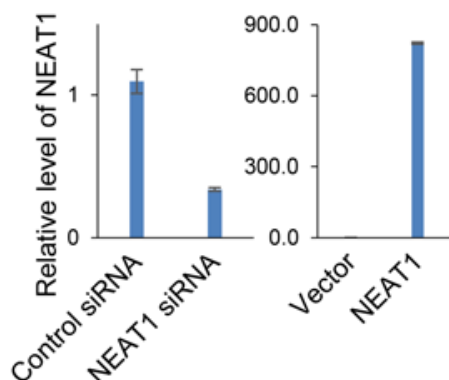

D
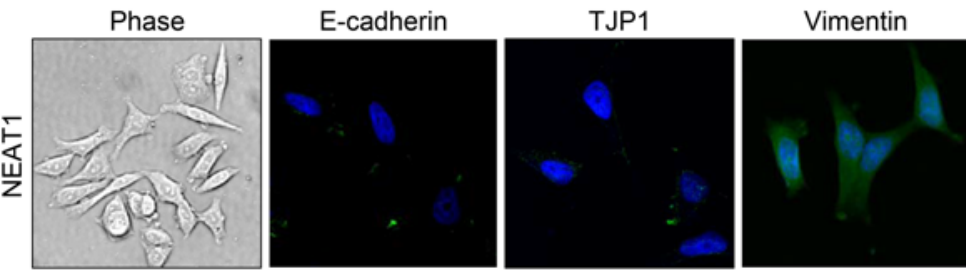

Fibronectin
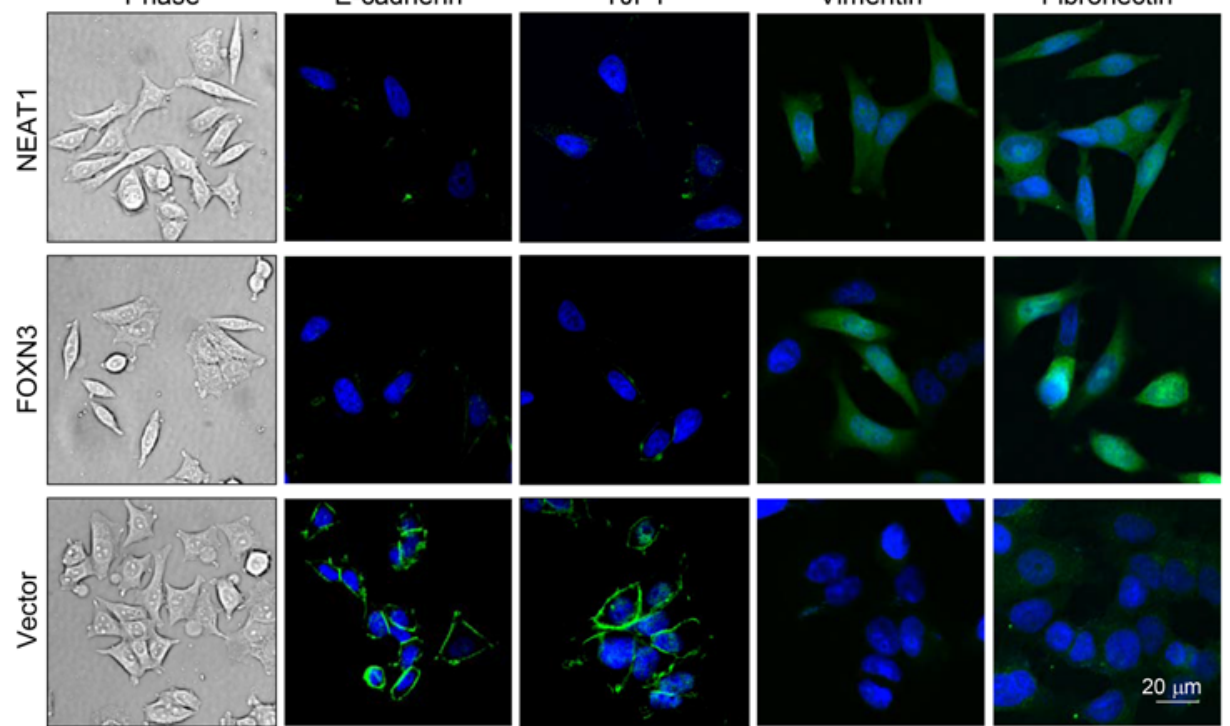

Figure 5. The FOXN3-NEAT1-SIN3A complex promotes EMT. (A) MCF-7 cells were transfected with FOXN3 or NEAT1 for the measurement of the indicated epithelial/mesenchymal markers by qPCR or Western blotting. (B) MCF-7 cells were transfected with FOXN3 siRNA or NEAT1 siRNA for the measurement of the indicated epithelial/mesenchymal markers by qPCR or Western blotting. (C) MCF-7 cells were transfected with the indicated siRNAs and/or expression vectors for the measurement of the indicated epithelial/mesenchymal markers by qPCR. The efficiency of knockdown or overexpression was verified by Western blotting or qPCR. In A-C, error bars represent mean \pm SD for triplicate experiments ( $P$ < 0.05 , 2 -way ANOVA). (D) MCF-7 cells were transfected with FOXN3 or NEAT1 for the morphological examination by phase-contrast microscopy. The epithelial (E-cadherin and TJP1) and mesenchymal (vimentin and fibronectin) markers were immunofluorescently stained and analyzed by confocal microscopy. DAPI staining was included to visualize the nucleus (blue). Representative images from triplicate experiments are shown. 
SAP18 is an integral component of the FOXN3-SIN3A complex, IP with antibodies against SAP18 followed by IB with antibodies against SIN3A, HDAC1, HDAC2, and FOXN3 detected the interaction of SAP18 with FOXN3-SIN3A complex in cellular lysates from MCF-7 cells (Figure 4E). RNA pull-down assays were then performed with GST/His double-tagged SAP18 (GST/ His-SAP18) or SAP18 mutants with potential RBRs deleted (GST/ His-SAP18 $\Delta 17-29$, GST/His-SAP18 $\Delta 139-149)$ and an in vitrotranscribed and biotinylated fragment of NEAT1 (704-903 nt) that was bound to streptavidin-conjugated agarose resin. Western blotting showed that while GST/His-SAP18 and GST/HisSAP18 $139-149$ were capable of interacting with NEAT1, GST/ His-SAP18 $\Delta 17-29$ lost its ability to interact with NEAT1 (Figure $4 \mathrm{~F}$ ). These results not only support an interaction between SAP18 and NEAT1 but also suggest that the sequence 17-29 aa represents the potential RBR of SAP18.

To further support the proposition that NEAT1 is a facultative component of the SIN3A complex, FLAG-tagged SAP18 (FLAG-SAP18) or SAP18 mutants (FLAG-SAP18 17 -29, FLAGSAP18 $139-149)$ were transfected into MCF-7 cells for RIP-qPCR assays with anti-FLAG. Consistent with the results described above, the interaction of SAP18 with NEAT1 was abrogated when amino acids 17-29 of SAP18 were removed (Figure 4G, left). Significantly, RIP-qPCR experiments with anti-SIN3A in a FOXN3-knockout MCF-7 cell line that we established using the CRISPR/Cas9 system detected the enrichment of NEAT1 in SIN3A RIP in the absence of FOXN3 (Figure 4G, right). Together, these results support the proposition that NEAT1 is a facultative component of the SIN3A complex.

The FOXN3-NEAT1-SIN3A complex promotes EMT and invasion of breast cancer cells in vitro and the dissemination and metastasis of breast cancer in vivo. EMT is a hallmark of cancer and represents the initial step of tumor metastasis (36). The identification of GATA3 and tight junction protein 1 (TJP1 [ZO1]), one of the epithelial markers by itself, as the targets of the FOXN3-NEAT1SIN3A complex suggests that this complex might play an important role in the regulation of EMT and invasion of breast cancer cells. To test this, gain-of-function and loss-of-function of FOXN3 and NEAT1 experiments were performed in MCF-7 cells, and the expression of epithelial/mesenchymal markers was analyzed and the morphology of the cells was examined. We found that overexpression of either FOXN3 or NEAT1 resulted in a reduction, at both mRNA and protein levels, of epithelial markers including E-cadherin, $\alpha$-catenin, and $\gamma$-catenin and induction of mesenchymal markers including fibronectin and vimentin (Figure 5A). Consistently, depletion of FOXN3 or NEAT1 was associated with induction of epithelial markers and reduction of mesenchymal markers (Figure 5B). In addition, FOXN3 and NEAT1 were probably interdependent in regulating the expression of the epithelial/ mesenchymal markers, as knockdown of NEAT1 in FOXN3-overexpressing MCF-7 cells was associated with a diminished effect of FOXN3 on the alterations of the expression of the epithelial/mesenchymal markers, and knockdown of FOXN3 in NEAT1-overexpressing MCF-7 cells was accompanied by an abrogated effect of NEAT1 on the alterations of the levels of the epithelial/mesenchymal markers (Figure 5C). Moreover, the effect of FOXN3 or NEAT1 on the expression of these markers was probably dependent on the SIN3A complex, as knockdown of SIN3A in FOXN3- or NEAT1-overexpressing MCF-7 cells resulted in diminished effects of FOXN3 and NEAT1 (Figure 5C). Furthermore, the effect of FOXN3 or NEAT1 on the expression of the epithelial/mesenchymal markers was, at least partially, through downregulation of GATA3, as simultaneous overexpression of GATA3 in FOXN3- or NEAT1-overexpressing MCF-7 cells counteracted the alterations of the expression of epithelial/mesenchymal markers and concomitant knockdown of GATA3 in FOXN3- or NEAT1-depleted MCF-7 cells was associated with a reversed trend in the alteration of the expression of epithelial/mesenchymal markers (Figure 5C).

Morphologically, while control cells maintained organized cell-cell adhesion and cell polarity, FOXN3- or NEAT1-overexpressing cells exhibited loss of cell-cell contacts; cells became scattering and their cobblestone-like appearance was replaced by a spindle-like, fibroblastic morphology, indicative of characteristic morphological changes of EMT (Figure 5D). In agreement with these observations, immunostaining showed a reduced or lost staining of the epithelial markers E-cadherin and TJP1 in FOXN3or NEAT1-overexpressing MCF-7 cells, while the mesenchymal markers vimentin and fibronectin displayed a reverse trend (Figure 5D). Together, these results are consistent with our proposition that the FOXN3-NEAT1-SIN3A complex, through transcription repression of downstream target genes including GATA3 and TJP1, promotes EMT.

We next investigated the role of the FOXN3-NEAT1-SIN3A complex in cellular behavior of breast cancer cells using Transwell invasion assays. As shown in Figure 6A, overexpression of either FOXN3 or NEAT1 resulted in a marked increase in the invasive potential of MCF-7 cells. In addition, FOXN3 and NEAT1 were probably interdependent in promoting cell invasion, as knockdown of NEAT1 in FOXN3-overexpressing MCF-7 cells was associated with a diminished effect of FOXN3 on cell invasion, and knockdown of FOXN3 in NEAT1-overexpressing MCF-7 cells abrogated the invasion-promoting effect of NEAT1 (Figure 6A). Moreover, the invasion-promoting effect of overexpression of FOXN3 or NEAT1 was probably dependent on SIN3A, as knockdown of SIN3A in FOXN3- or NEAT1-overexpressing MCF-7 cells abrogated the effects of FOXN3 and NEAT1 (Figure 6A). Furthermore, the invasion-promoting effect of overexpression of FOXN3 or NEAT1 was, at least partially, through transcription repression of GATA3, as simultaneous overexpression of GATA3 in FOXN3- or NEAT1-overexpressing MCF-7 cells was associated with reversed phenotypes (Figure 6A). Neither overexpression nor knockdown of FOXN3 had a significant effect on the invasive potential of MDA-MB-231 cells (Figure 6B). Collectively, these results suggest that the FOXN3NEAT1-SIN3A complex, through transcription repression of downstream target genes including GATA3, promotes cell invasion in hormonally responsive breast cancer.

To investigate the role of the FOXN3-NEAT1-SIN3A complex in breast cancer dissemination and metastasis in vivo, MCF-7 cells that had been engineered to stably express firefly luciferase (MCF7 Red-Luc, PerkinElmer) were infected with lentiviruses carrying empty vector/control shRNA, FOXN3, NEAT1, FOXN3+shSIN3A, NEAT1+shSIN3A, or FOXN3+GATA3. These cells were then implanted orthotopically onto the abdominal mammary fat pad of 6-week-old ovariectomized female immunocompromised severe combined immunodeficiency (SCID) mice implanted with E2 pel- 
A
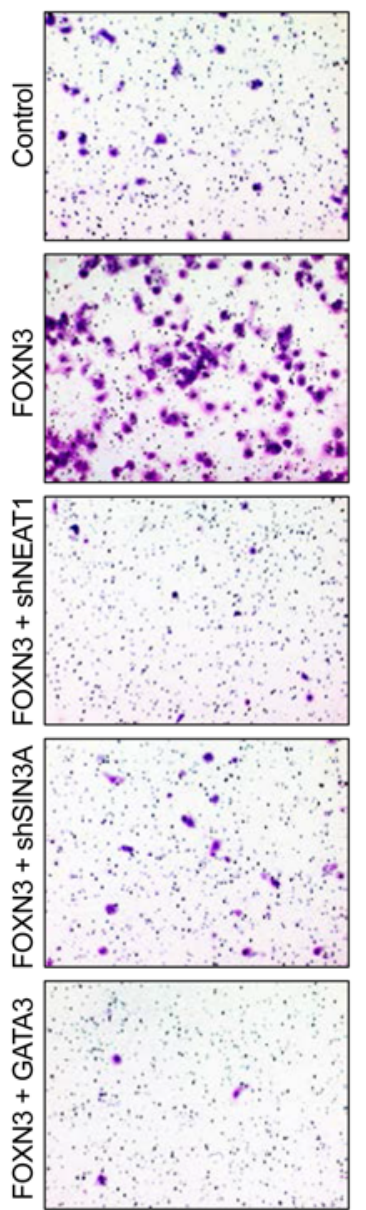

B
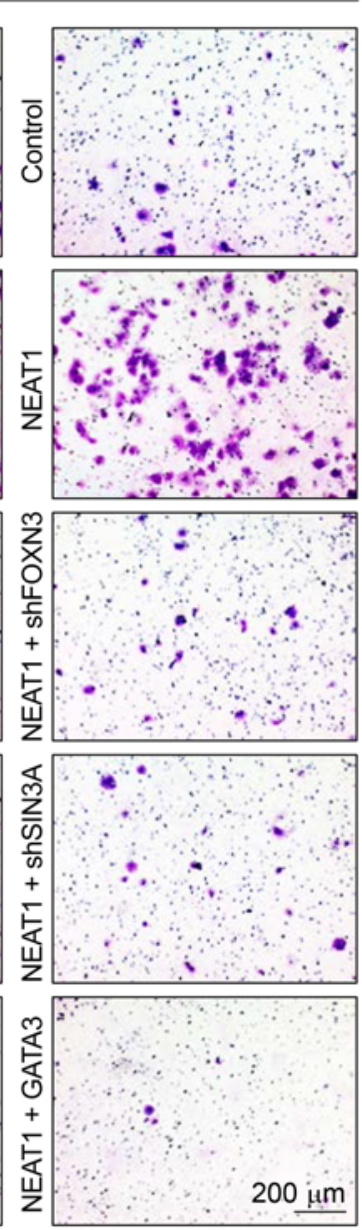
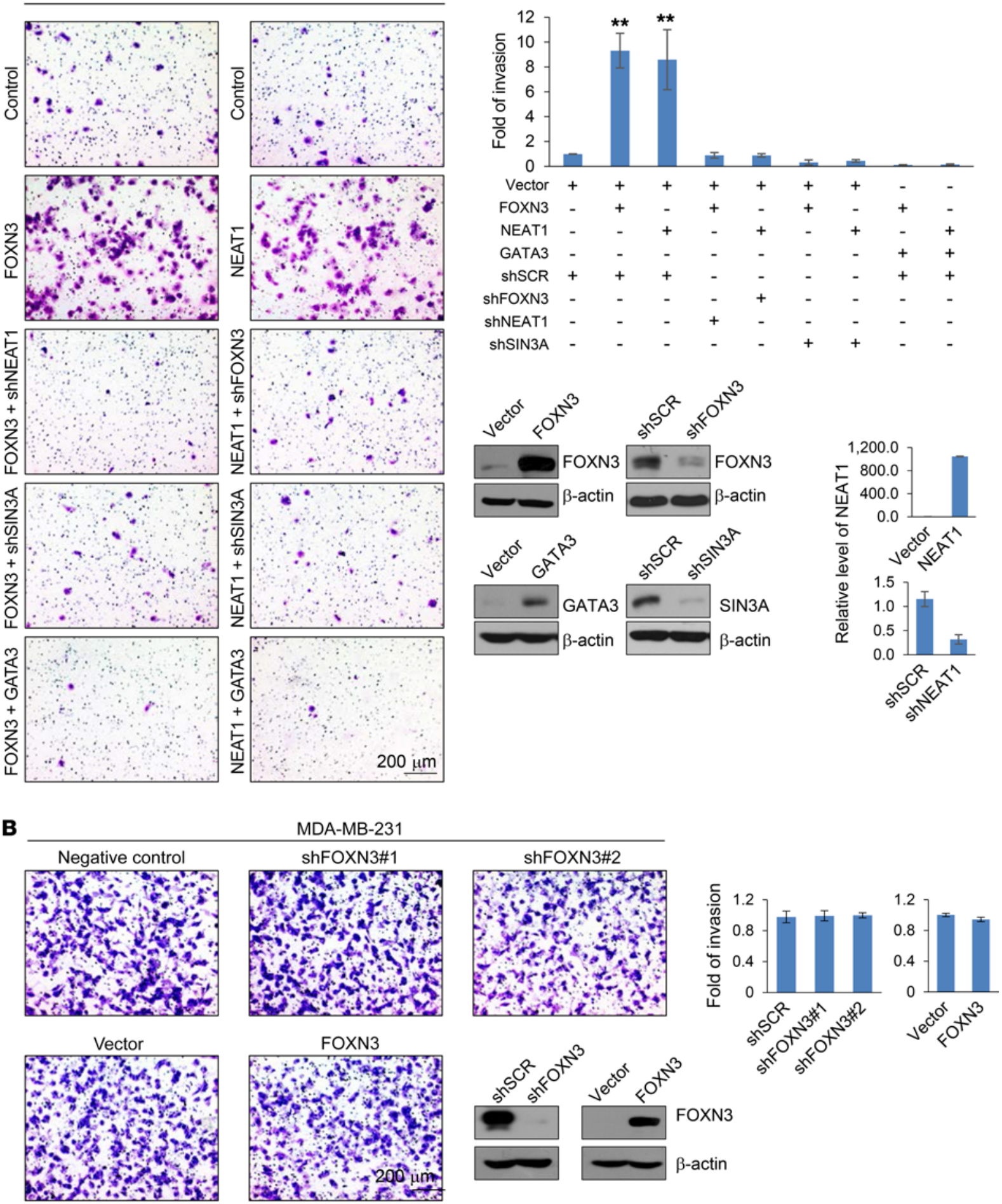

Figure 6. The FOXN3-NEAT1-SIN3A complex promotes breast cancer cell invasion. (A) MCF-7 cells were infected with lentiviruses carrying the indicated shRNAs and/or expression constructs for Transwell cell invasion assays. The invaded cells were stained and counted. The images represent 1 field under microscopy in each group. The efficiency of knockdown or overexpression was verified by Western blotting or qPCR. Error bars represent mean \pm SD for triplicate experiments ( ${ }^{* *} P<0.01$, 1-way ANOVA). (B) Transwell cell invasion assays in MDA-MB-231 cells as described in $\mathbf{A}$.

lets $(n=8)$. The growth of the primary tumor and metastasis of the otherwise nonmetastatic MCF-7 cells were monitored weekly and measured by quantitative bioluminescence imaging after 10 weeks with the IVIS imaging system (Xenogen). A metastatic event was defined as any detectable luciferase signal above background and away from the primary tumor site. The control MCF-7 cells basically generated no detectable metastases (Figure 7A). However, overexpression of either FOXN3 or NEAT1 effectively promoted lung 


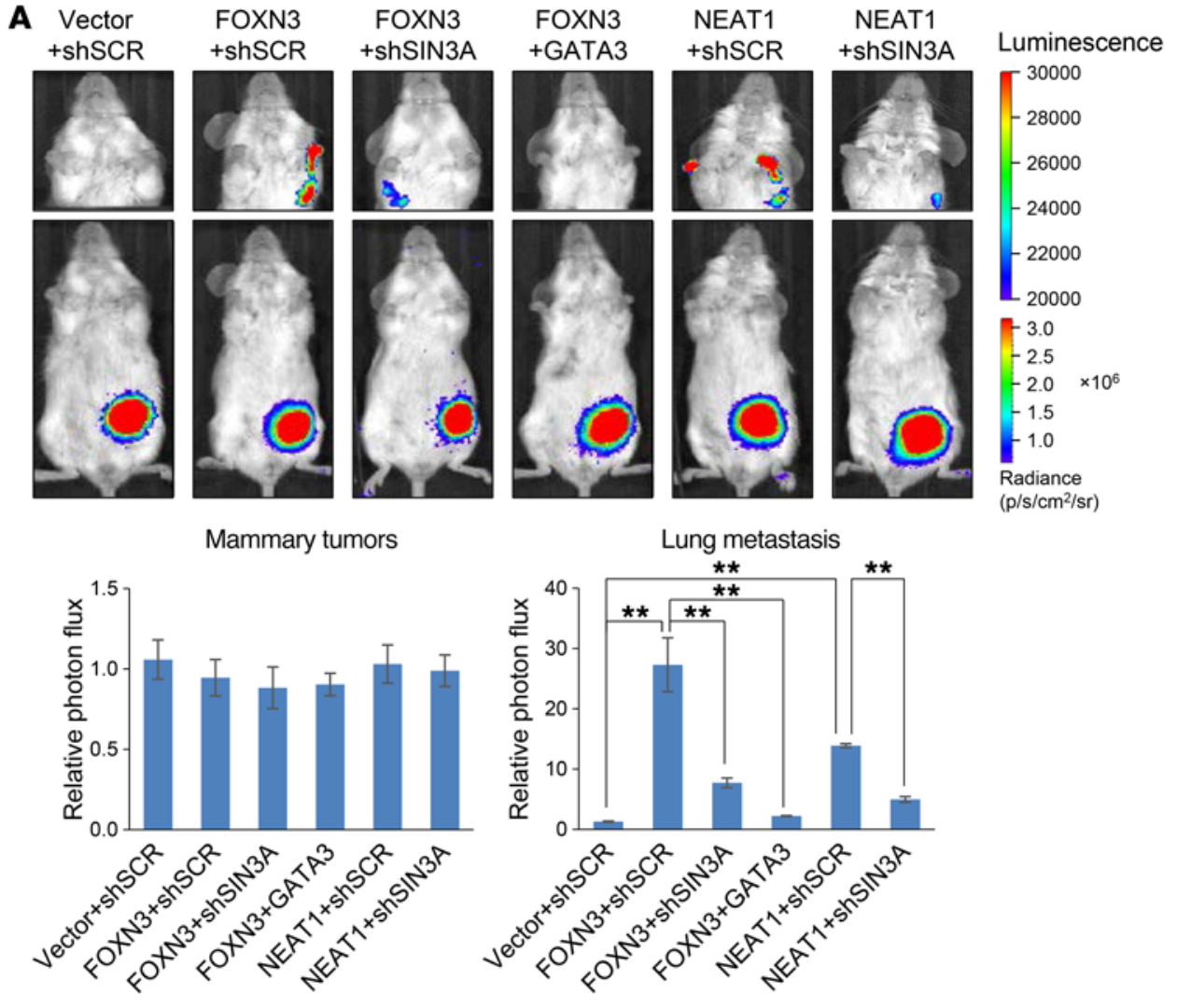

B

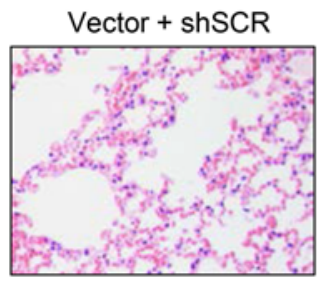

FOXN3 + GATA3

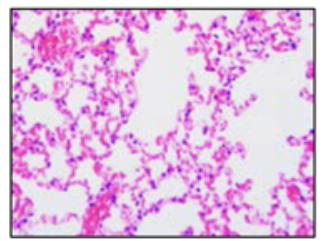

Lung metastasis (H\&E)

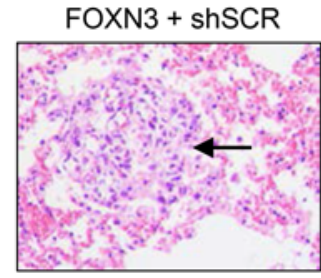

NEAT1 + shSCR

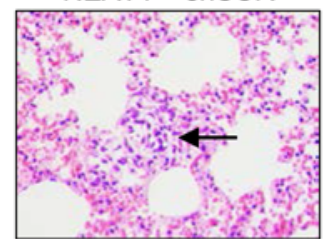

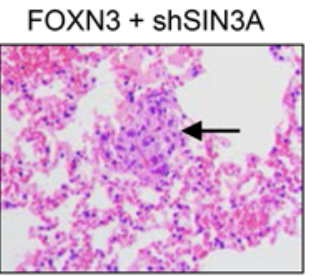

NEAT1 + shSIN3A

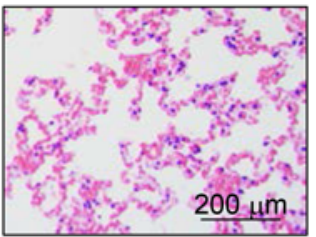

Figure 7. The FOXN3-NEAT1-SIN3A complex promotes breast cancer metastasis. (A) MCF-7 Red-Luc cells infected with lentiviruses carrying the indicated expression constructs and/or shRNAs were inoculated orthotopically into the abdominal mammary fat pad of 6 -week-old female SCID mice $(n=8)$. Primary tumors and metastases were quantified using bioluminescence imaging after 10 weeks of initial implantation. Representative in vivo bioluminescent images are shown. Error bars represent mean \pm SEM ( ${ }^{* *} P<0.01,1$-way ANOVA). (B) Lung metastases were examined by $\mathrm{H} \& \mathrm{E}$ staining. p, photon flux; sr, steradian.

metastasis of the MCF-7 Red-Luc tumors (Figure 7A). In addition, the lung metastasis-promoting effect of FOXN3 or NEAT1 was probably through the SIN3A complex, as depletion of SIN3A in FOXN3- or NEAT1-overexpressing tumors led to a diminished effect of FOXN3 and NEAT1 (Figure 7A). Moreover, the metastasis-promoting effect of overexpression of FOXN3 was, at least partially, through down-

regulation of GATA3, as simultaneous overexpression of GATA3 in the FOXN3-overexpressing group was associated with a diminished lung metastasis (Figure 7A). The lung metastases were verified by histological staining (Figure 7B). Although several of the above experiments were based on the overexpression of FOXN3 and NEAT1, these experiments nevertheless support a potential role for the FOXN3-NEAT1SIN3A complex in promoting breast cancer metastasis.

Negative-feedback regulation of estrogen response by the FOXN3-NEAT1SIN3A complex. Our ChIP-Seq and CHART-Seq analysis revealed that $\mathrm{ER} \alpha$ is also a transcriptional target of the FOXN3-NEAT1-SIN3A complex. Indeed, FOXN3, SIN3A, and NEAT1 had similar peak distributions on ESR1 (Figure 8A). Given our observations that NEAT1 is induced by estrogen and participates in transcription regulation by the FOXN3-NEAT1-SIN3A complex, transcriptional targeting of ER $\alpha$ by the FOXN3-NEAT1-SIN3A complex implies that there exists a negative-feedback loop in $\mathrm{ER} \alpha^{+}$breast cancer cells. To test this, we first verified the transcription repression of ER $\alpha$ by the FOXN3-NEAT1-SIN3A complex. To this end, MCF-7 cells were transfected with vector/control siRNA, FOXN3, FOXN3+SIN3A siRNA, or FOXN3+NEAT1 siRNA, and the expression of ER $\alpha$ was measured by qPCR and Western blotting. The results showed that overexpression of FOXN3 was associated with a decrease in ER $\alpha$ expression, but only in the presence of SIN3A and NEAT1; depletion of either SIN3A or NEAT1 led to a diminished effect of FOXN3 overexpression (Figure 8B). Consistently, qChIP detected the binding of FOXN3 and SIN3A on the $-1,500$ to -590 region of the ESR1 promoter, but not in a control region (Figure 8C). Further qPCR analysis showed that overexpression of FOXN3 was associated with a decrease in the expression of the estrogen-responsive genes TFF1 and GREB1, while depletion of either SIN3A or NEAT1 abrogated the effect (Figure 8D). Together, these results indicate that ER $\alpha$ is regulated by the FOXN3NEAT1-SIN3A complex, supporting the existence of a negative-feedback loop between ER $\alpha$ and the FOXN3-NEAT1-SIN3A complex. 
A

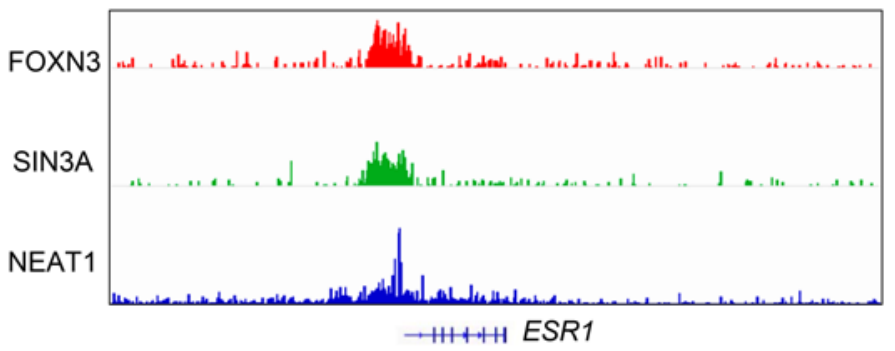

B

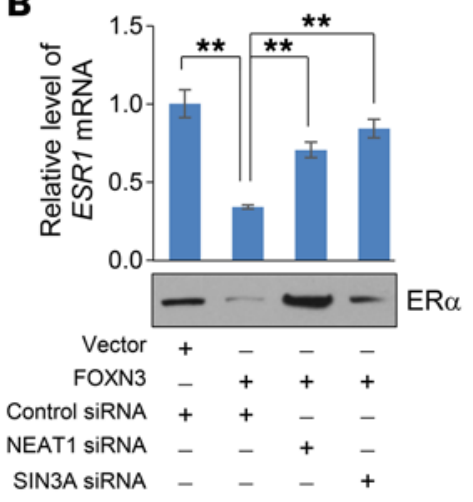

C

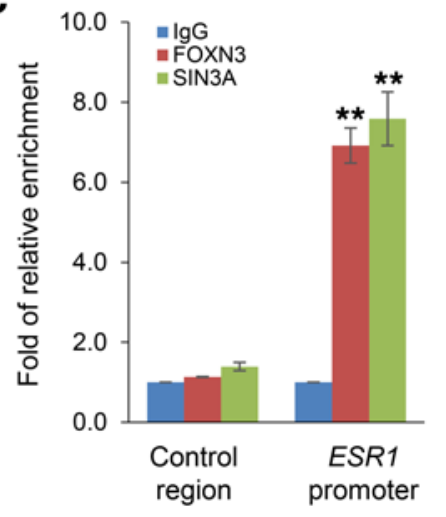

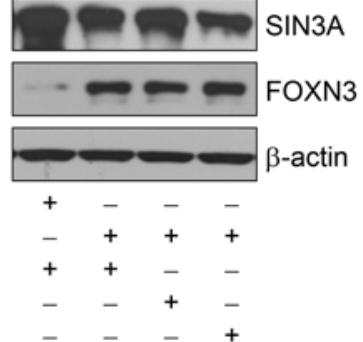

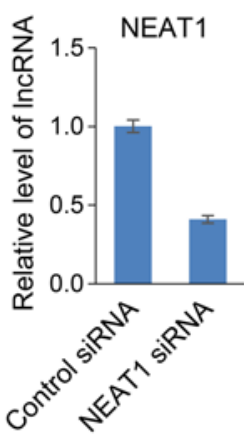

D

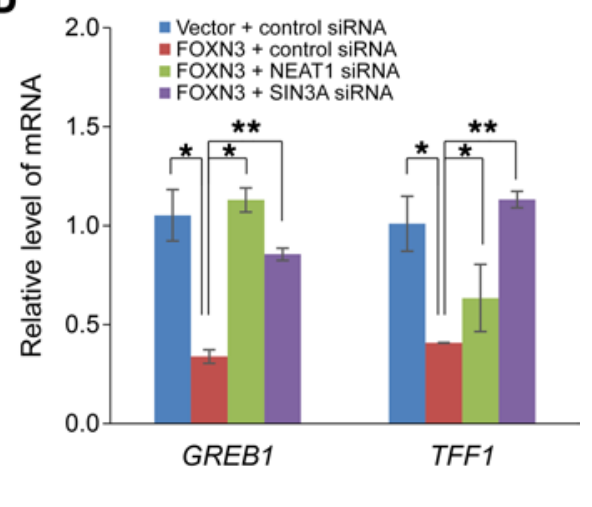

Figure 8. Negative-feedback regulation of estrogen response by the FOXN3-NEAT1-SIN3A complex. (A) The binding profiles of FOXN3, SIN3A, and NEAT1 on ESR 1 based on ChIP-Seq and CHART-Seq data. (B) MCF-7 cells were transfected with the indicated expression constructs and/or siRNAs for the measurement of ER $\alpha$ expression by qPCR and Western blotting. The efficiency of knockdown or overexpression was verified by Western blotting or GPCR. Error bars represent mean \pm SD for triplicate experiments (**P $<0.01,1$-way ANOVA). (C) qChIP analysis of the promoter of ESR1 or a control region in MCF-7 cells for the binding of FOXN3 and SIN3A. (D) MCF-7 cells were transfected with the indicated expression vectors and/or siRNAs for the measurement of the expression of GREB1 and TFF 1 by qPCR. In $\mathbf{C}$ and $\mathbf{D}$, error bars represent mean \pm SD for triplicate experiments $\left({ }^{*} P<0.05,{ }^{*} P<0.01 ; 2\right.$-way ANOVA).

(Figure 9B). Meanwhile, immunohistochemical staining revealed that GATA3 is downregulated in breast carcinoma samples and the level of its expression is negatively correlated with the histological grades of the tumors (Figure 9B).

To further extend our observations to clinicopathologically relevant settings, analysis of a public data set (GSE27562) also revealed that both FOXN3 $\left(P=7.57 \times 10^{-4}\right)$ and NEAT1 $(P=0.01839)$ are upregulated in breast cancer (Figure 10A, left). Interrogation of Bittner's breast cancer data set in Oncomine (https:// www.oncomine.org/) also showed that the levels of both FOXN3 and NEAT1 are positively correlated with the histological grades of $\mathrm{ER}^{+}$ breast cancer (Figure 10A, middle). Querying a public data set (GSE21653) revealed that, remarkably, high levels of FOXN3 $(P=0.005773)$ and NEAT1 $(P=0.008998)$

FOXN3 and NEAT1 are upregulated in breast cancer and their high levels are correlated with higher tumor grades and worse survivals. To further support the role of the FOXN3-NEAT1-SIN3A complex in breast cancer progression and to extend our observations to clinicopathologically relevant contexts, we collected 24 breast carcinoma samples paired with adjacent normal mammary tissues from breast cancer patients and analyzed by qPCR for the expression of FOXN3 and NEAT1. The results showed that both FOXN3 and NEAT1 are upregulated in breast cancer (Figure 9A). In addition, consistent with our working model that the FOXN3-NEAT1-SIN3A complex transcriptionally represses GATA3, when the relative expression level of GATA3 was plotted against that of FOXN3 or NEAT1, significant negative correlations were found (Figure 9A).

We then analyzed the protein level of FOXN3 by immunohistochemical staining of a human tissue array containing 19 breast carcinoma samples paired with normal mammary tissues as well as tissue arrays including 15 normal mammary tissues and 160 breast carcinoma samples from patients with grade I (26), grade II (82), or grade III (52) breast cancer. We found that FOXN3 is upregulated in breast carcinoma samples, and the level of its expression is positively correlated with the histological grades of the tumors expression in $\mathrm{ER}^{+}$breast carcinomas are strongly correlated with the lymph node positivity of the patients (Figure 10A, right). No statistically significant correlations with the histological grades and the lymph node positivity were found in ER- breast cancer (Figure 10B). Further analysis of a public data set (GSE48390) showed statistically significant negative correlations of the expression between FOXN3 and GATA3 $(P=0.00509)$ and between NEAT1 and GATA3 $(P=0.03129)$ in $\mathrm{ER}^{+}$breast cancer, whereas in ER breast cancer, no such correlations were found (Figure 10C). In addition, statistically significant negative correlations of the expression between FOXN3 and GATA3 $\left(P=2.35 \times 10^{-8}\right)$ and between NEAT1 and GATA3 $\left(P=4.92 \times 10^{-6}\right)$ were also found in a data set (GSE27652) with unknown ER status (Figure 10C). Moreover, in support of our observation that NEAT1 is induced by estrogen, analyses of Tabchy's, Lu's, Esserman's, and Desmedt's breast cancer data sets in Oncomine (https://www.oncomine.org/) indicate that the expression level of NEAT1 is significantly higher in $\mathrm{ER}^{+}$breast cancer than in $\mathrm{ER}^{-}$breast cancer (Figure 11A).

Finally, to substantiate the clinicopathological significance of our observations, we analyzed the expression levels of FOXN3, NEAT1, and SIN3A in breast cancer and their correlations with 

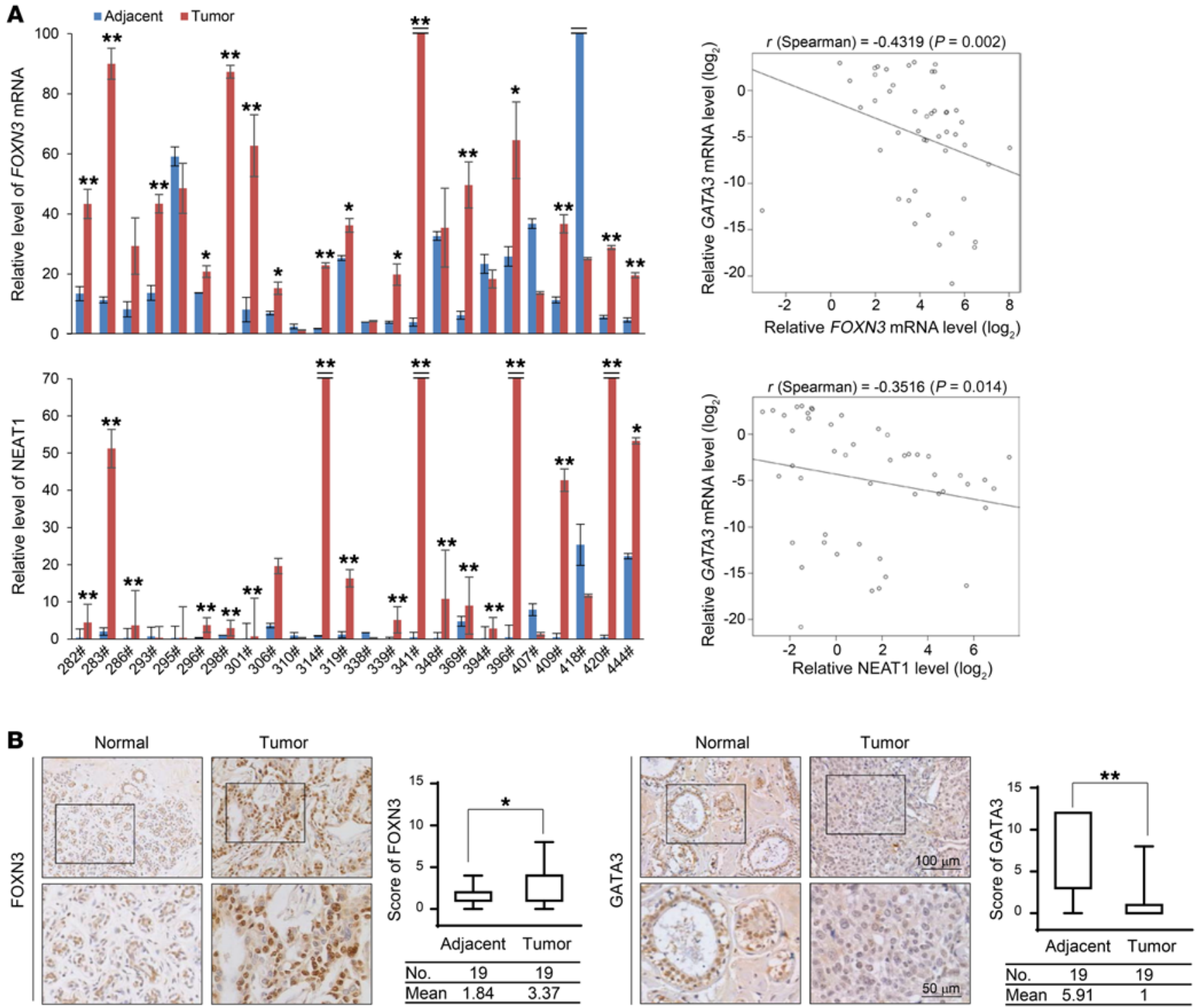

Figure 9. The expression of FOXN3 and NEAT1 in breast cancer. (A) qPCR analysis of 24 paired breast carcinoma samples for the expression of FOXN3, NEAT1, and GATA3. The relative level of GATA3 was plotted against that of FOXN3 or NEAT1. Each bar represents mean \pm SD for triplicate experiments $\left({ }^{*} P<0.05\right.$, ${ }^{* *} P<0.01 ; t$ test). (B) Tissue array analysis of 19 paired breast carcinoma samples $\left({ }^{*} P<0.05,{ }^{*} P<0.01\right.$; $t$ test) (top) or 15 normal mammary tissues plus 160 breast carcinoma samples (grades I, II, and III) for the expression of FOXN3 and GATA3 $\left({ }^{*} P<0.05\right.$, ${ }^{* *} P<0.01 ; 1$-way ANOVA) (bottom). Representative images are shown. The positively stained nuclei were analyzed, and the mean staining intensity was scored by Image-Pro Plus software (Media Cybernetics).
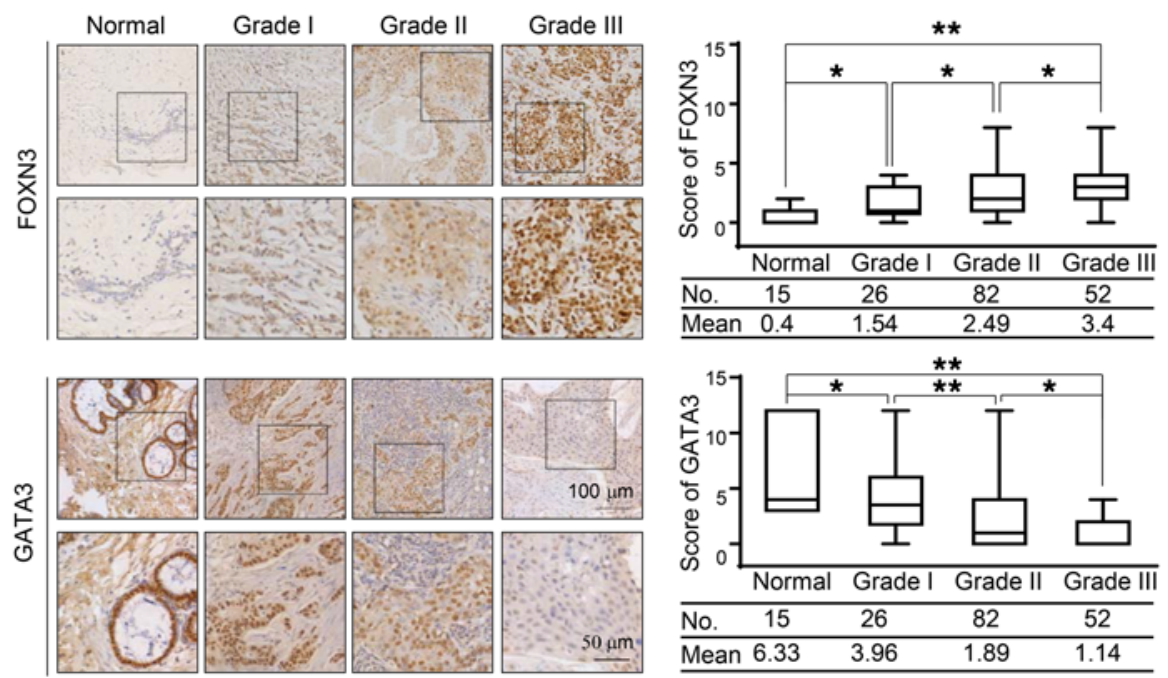
A
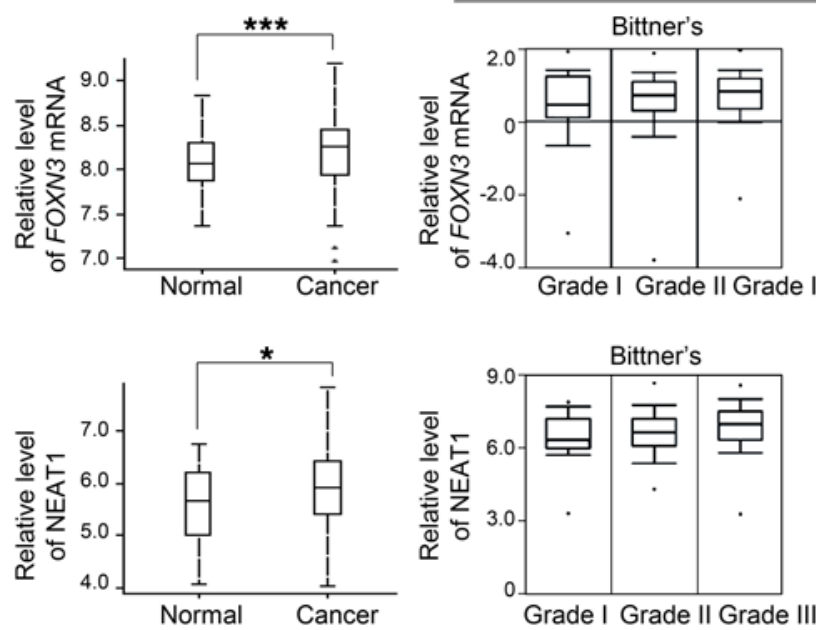

B

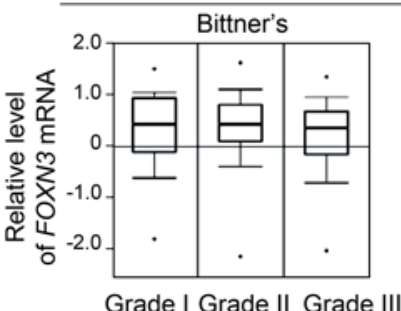

ER-
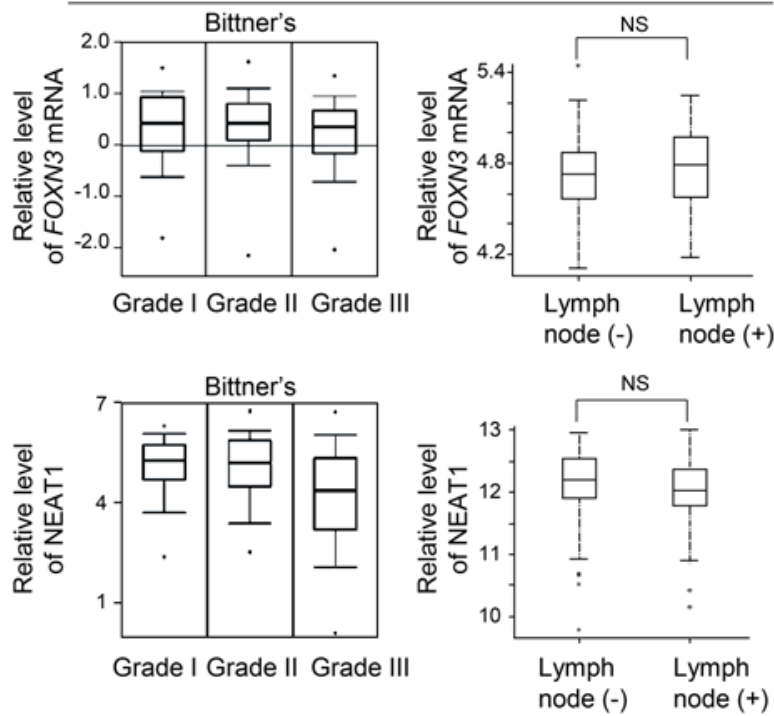

Figure 10. The expression of FOXN3 and NEAT1 in breast cancer and their association with the histological grades and lymph node positivity of the tumors. (A) Bioinformatics analysis of the public data set (CSE27562) in breast carcinoma samples and normal mammary tissues (left), Bittner's breast cancer data set in Oncomine (middle), or the public data set (CSE21653) for the expression of FOXN3 and NEAT1 based on the indicated stratifications (right). ${ }^{*} P<$ $0.05,{ }^{* *} P<0.01,{ }^{* *} P<0.001 ; t$ test. (B) Analysis of Bittner's breast cancer data set in Oncomine (left) or the public data set (CSE21653) for the expression of FOXN3 and NEAT1 based on the indicated stratifications (right). (C) Analysis of public data sets (CSE48390, GSE27562) for the expression of FOXN3, NEAT1, and GATA3. The relative level of GATA3 was plotted against that of FOXN3 or NEAT1.

$\mathrm{ER}^{+}$
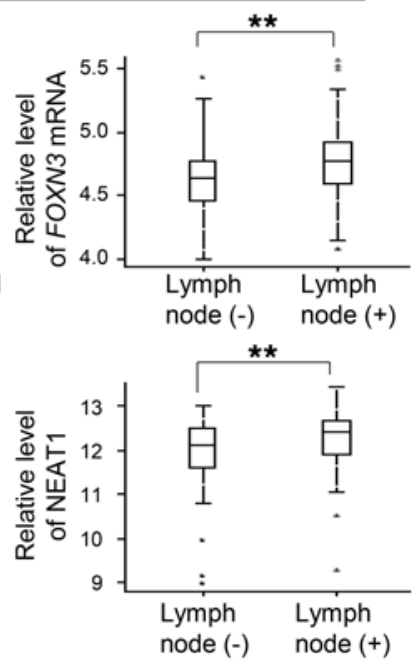

C

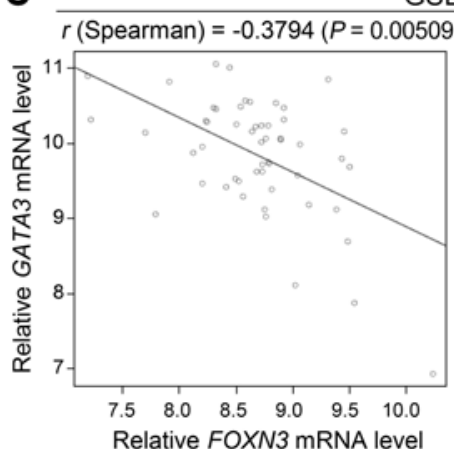

GSE48390
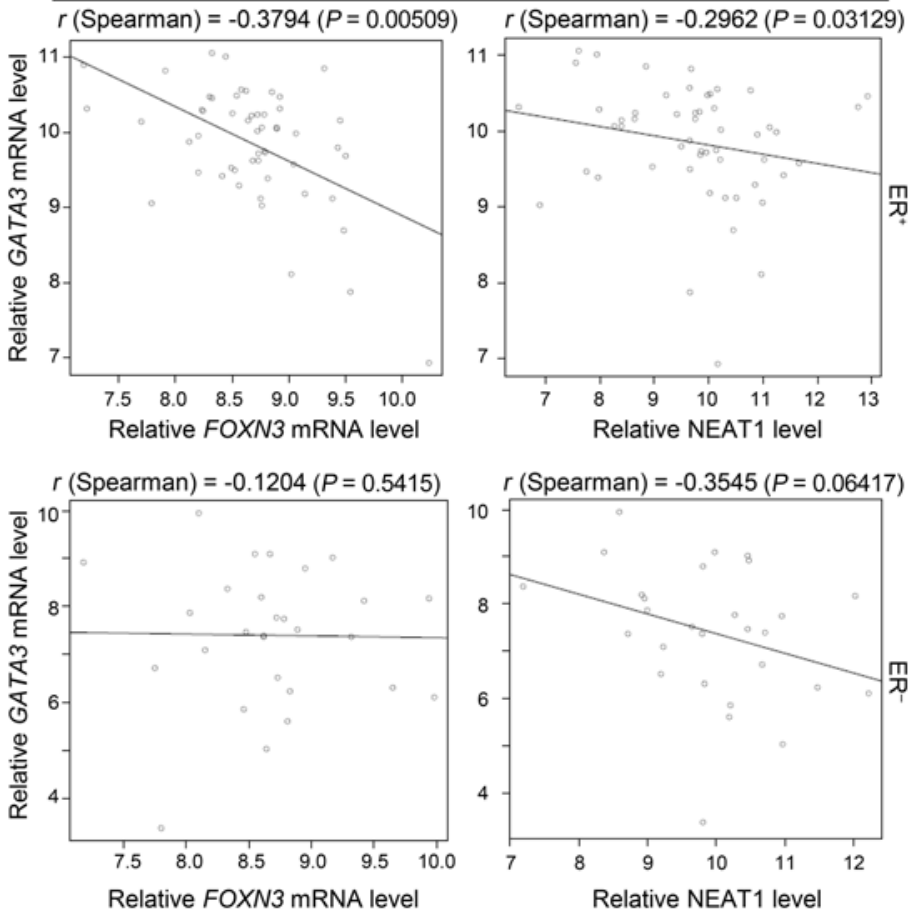

GSE27562
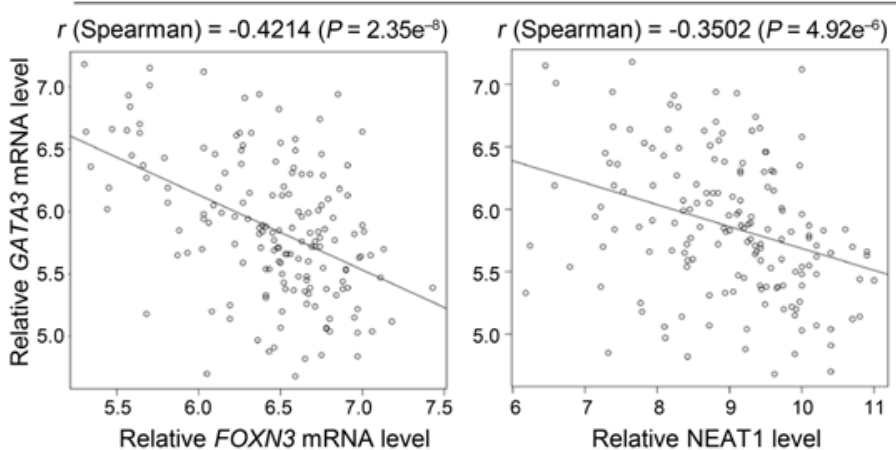
A

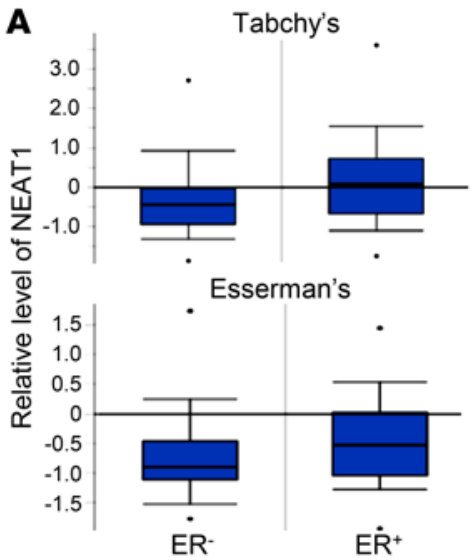

B
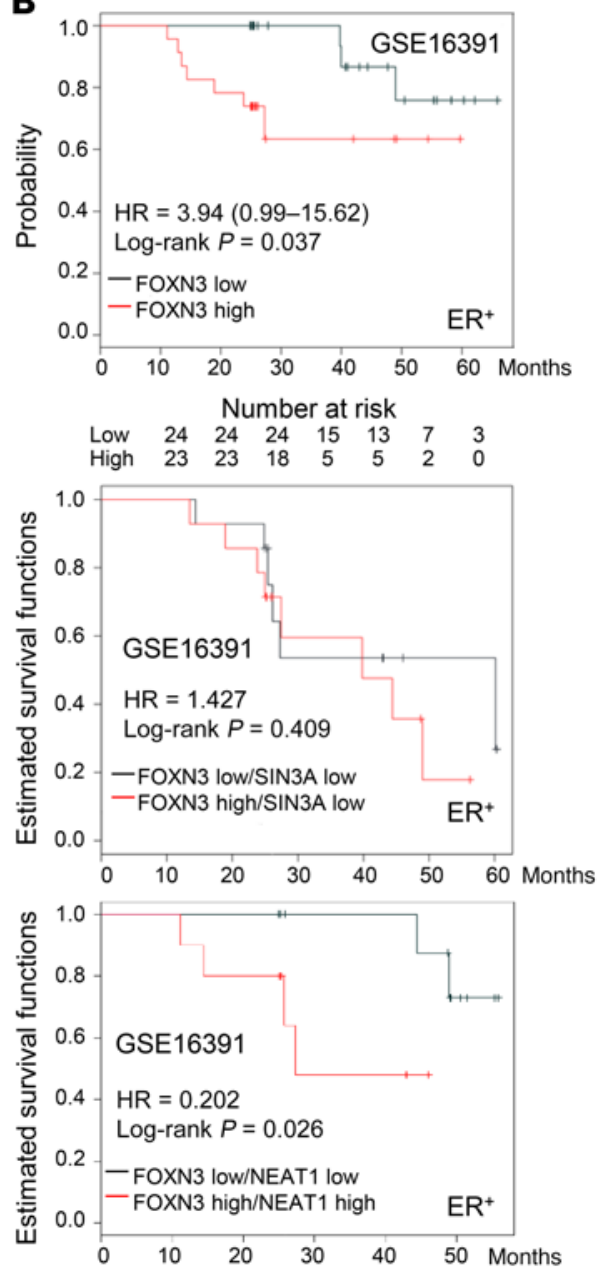
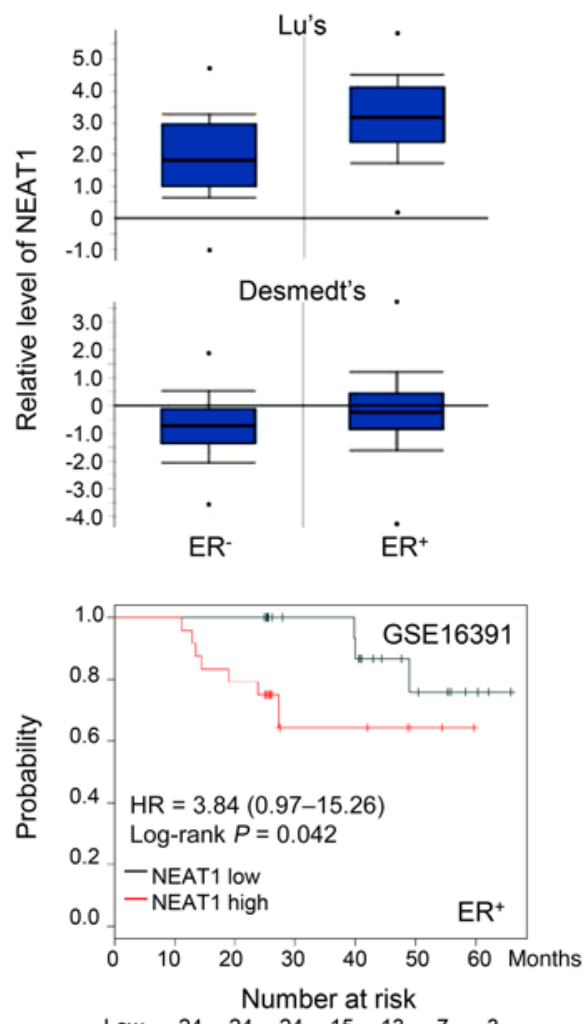
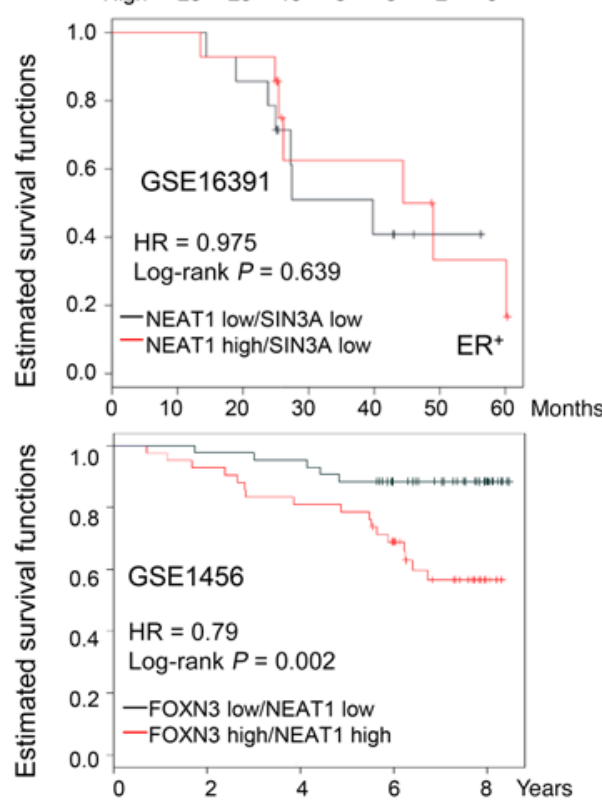

C

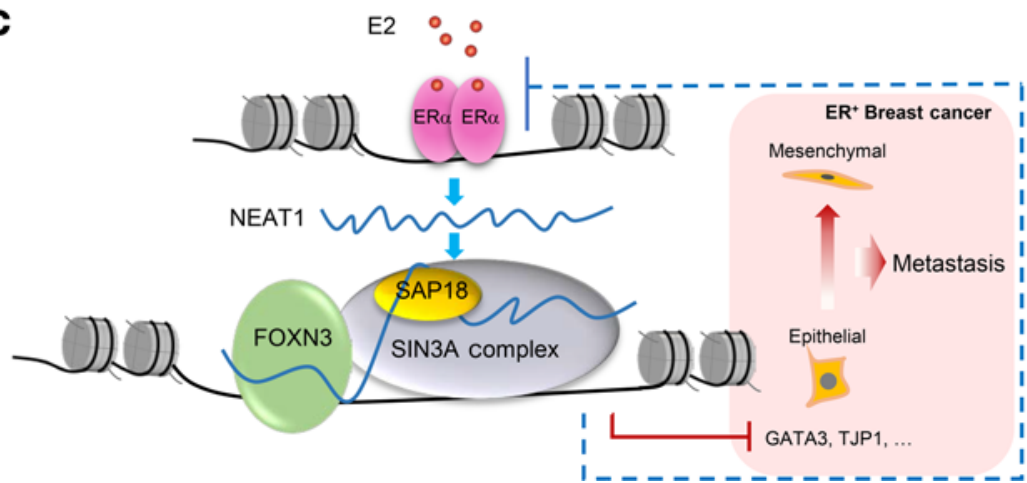

Figure 11. The expression of FOXN3 and NEAT1 in breast cancer and their association with clinical behaviors. (A) Analysis of the indicated Oncomine data sets for the expression of NEAT1 based on the indicated stratifications. (B) Kaplan-Meier survival analysis of the public data sets for the relationship between survival time of breast cancer patients with ER+ (GSE16391) or unknown ER status (GSE1456) and the expression of FOXN3, NEAT1, and/or SIN3A in breast cancer (by log-rank). HR, hazard ratio. (C) The proposed model of the ER $\alpha$ NEAT1-FOXN3/NEAT1/SIN3A-GATA3 axis in $\mathrm{ER}^{+}$breast cancer metastasis.

clinical behaviors of breast cancer patients. Kaplan-Meier survival analysis (http://kmplot.com/analysis/) of public data sets (GSE16391) showed that high expression of either FOXN3 ( $P=$ $0.037)$ or NEAT1 $(P=0.042)$ was associated with a worse overall survival of $\mathrm{ER}^{+}$breast cancer patients. However, when expression of SIN3A was low, high expression of FOXN3 $(P=0.409)$ or NEAT1 $(P=0.639)$ had no significant correlations with the overall survival of $\mathrm{ER}^{+}$breast cancer patients. Concomitant high levels of FOXN3 and NEAT1 significantly improved the predictive capability (GSE16391, $P=0.026)$. Even in a data set (GSE1456) with unknown ER status, concomitant high expression levels of FOXN3 and NEAT1 were strongly associated with a worse overall survival of breast cancer patients $(P=0.002)$ (Figure 11B). Collectively, the above observations are consistent with a role of FOXN3 in breast cancer progression by recruiting the NEAT1-containing SIN3A complex and targeting the downstream genes including GATA3.

\section{Discussion}

It has been reported that FOXN3 acts in both transcription repression (3, 8-10) and activation (11). We report in this study that FOXN3 acts as a transcription repressor and physically interacts with the SIN3A complex in human cells. Whether transcription activation represents an organism/lineage-specific mechanism for FOXN3 action is currently unknown, and how FOXN3 might coordinate transcription repression programs through interacting with distinct corepressor com- 
plexes remains an issue for future investigation. Nevertheless, our observations are consistent with the reports that FOXN3 interacts with $\mathrm{xSin} 3 / \mathrm{xRPD} 3$ in Xenopus laevis (3) and $\operatorname{Sin} 3 \mathrm{~A}$ in Saccharomyces cerevisiae (8), suggesting that the association with the SIN3A complex represents an evolutionarily conserved mechanism for the action of FOXN3 in animal development. Interestingly, we found that the interaction of FOXN3 with the SIN3A complex is only detected in $\mathrm{ER}^{+}$breast cancer cells and in the absence of RNase. Inspired by the findings that several $\operatorname{lncRNAs}$ are involved in protein-protein interactions $(32,33)$, we identified, by iRIP-Seq, that lncRNA NEAT1 is required for the interaction.

NEAT1 is an essential structural component of the nuclear paraspeckles (22). Although their function at the whole-animal level remains obscure, paraspeckles are mammalian-specific nuclear bodies built on NEAT1 and its associated proteins to control several biological processes including cellular differentiation and stress responses (42). As it is becoming increasingly clear that lncRNAs represent key components for the epigenetic regulatory network $(20,21)$, a transcriptional regulatory activity for NEAT1 is also expected. Indeed, a recent study suggests that NEAT1 is capable of influencing gene transcription indirectly by sequestration of a transcriptional regulator in paraspeckles (23), and, remarkably, it is demonstrated that NEAT1 is also able to bind to genomic sites in human cells (24), implying direct transcription regulation by NEAT1. We propose that NEAT1 is a facultative component of the SIN3A complex that is recruited by FOXN3 to chromatin to negatively regulate transcription, providing a molecular mechanism for transcriptional regulation by NEAT1 and supporting an argument that NEAT1 is another important epigenetic regulator.

The notion that NEAT1 is an architectural determinant of the nuclear paraspeckles yet functions as a transcription regulator is certainly puzzling. After all, paraspeckles and chromatin are allocated in different nuclear domains. However, it is worth noting that the gene encoding NEAT1 produces, via alternative 3 '-end processing, 2 transcripts, NEAT1_1 and NEAT1_2, and that it is believed that NEAT1_2 is the NEAT1 isoform required for the formation of the nuclear paraspeckles (43). Although our iRIP-Seq could not distinguish NEAT1_1 and NEAT1_2, the sequences identified in iRIP-Seq were predominantly matched to the NEAT1 5 '-terminal region, favoring an idea that the NEAT1 subspecies involved in the interaction with the FOXN3-SIN3A complex is NEAT1_1. Thus, it is a distinct possibility that different NEAT1 isoforms are involved in different nuclear activities. In this regard, it is interesting that our analysis of the molecular evolution of NEAT1 indicates that this gene arose only in vertebrates and is absent in Xenopus and yeast. Strikingly, this evolutionary scheme is concomitant with that of nuclear paraspeckles in that these nuclear organizations also emerged only from vertebrates (42). Apparently, an intrinsic link between NEAT1 and paraspeckles is beyond question. It is, however, conceivable that with the appearance of NEAT1 and the nuclear paraspeckles in vertebrates, they had to find a way to communicate with chromatin, and that that is why and when NEAT1_1 was born. Whether or not NEAT1_1 evolved later is currently unknown. At least at the molecular level, NEAT1_2 seems to be the parental form, with 22,743 bp in length, whereas NEAT1_1 is only 3,756 bp.
Future investigations will surely provide more accurate accounts on the evolution and function of the nuclear paraspeckles in relation to chromatin and transcription, especially in the context of NEAT1.

The question then is how FOXN3 interacts with SIN3A in Xenopus and yeast, which are without NEAT1, and why the interaction requires NEAT1 in vertebrates. It is logical to postulate that NEAT1 contributes to the stabilization of the FOXN3-SIN3A complex, especially in the chromatin context. It is also possible that NEAT1, in addition to its structural determination, contributes to specification of the genomic landscape of the FOXN3-SIN3A complex, thereby expanding or restricting the genomic targets of this complex in vertebrates. This kind of scheme is compatible with the organismal complexity and sophisticated gene regulatory network in vertebrates.

The SIN3A complex is a multiprotein assembly (44). Despite its well-recognized role in transcription repression, the SIN3A complex contains a panel of protein components whose functions in this complex remain enigmatic. Although our study indicates that one of these proteins, SAP18, is an RNA-binding protein capable of interacting with NEAT1, the inclusion of NEAT1 in the SIN3A complex further complicates the understanding of this assembly. Moreover, our proposal that NEAT1 is a facultative component of the SIN3A complex means that the NEAT1-SIN3A complex could be recruited by, in addition to FOXN3, other transcription repressors in other cell lineages. Future investigations are needed to identify the transcription factors interacting with the NEAT1-SIN3A complex and to delineate the cellular environment fostering the interaction. At least in our current study, we showed that the interaction between FOXN3 and the SIN3A complex occurs only in $\mathrm{ER}^{+}$breast cancer cells, where NEAT1 is induced by estrogen, consistent with a previous report that NEAT1 is an ER $\alpha$ target in prostate cancer (25).

Our ChIP-Seq experiments together with the analysis of NEAT1 CHART-Seq results (24) revealed that the FOXN3NEAT1-SIN3A complex represses a collection of genes including GATA3. GATA3 is unique in that it is the most highly expressed transcription factor in the mammary epithelium and its expression is restricted to the luminal epithelial cell population, where it not only specifies but also maintains luminal epithelial cell differentiation $(37,45)$. Thus, transcription repression of GATA3 by the FOXN3-NEAT1-SIN3A complex suggests an important role for FOXN3 in mammary gland development. Future investigations are needed to define the magnitude and multitude of FOXN3 and the FOXN3-NEAT1-SIN3A complex in animal development.

In recent years, both FOXN3 and NEAT1 have been implicated in malignancies of a broad spectrum of tissue origins. However, surprisingly, little is known about the role of FOXN3 and NEAT1 in the development and progression of breast cancer, and the mechanistic insights into their roles in other tumors are also limited. We showed in this study that the FOXN3-NEAT1-SIN3A complex, through transcription repression of downstream target genes including GATA3 and TJP1, promotes EMT and invasion of breast cancer cells in vitro and dissemination and metastasis of breast cancer in vivo. Intriguingly, we showed that ER $\alpha$ itself is a target of the FOXN3-NEAT1-SIN3A complex. Apparently, a feedback loop between ER $\alpha$ and the FOXN3-NEAT1-SIN3A complex exists in mammary tissue in which ER $\alpha$ transactivates NEAT1, which is assembled into the FOXN3-NEAT1-SIN3A complex, and 
this complex, in turn, transrepresses ER $\alpha$ (Figure 11C). As ER $\alpha$ is a classical etiological factor for breast cancer that is required for the growth of $E R \alpha^{+}$breast cancer cells (46), and as growth and differentiation are distinct and uncoupled cellular processes (47), it is conceivable that when mammary epithelial cells favor growth, NEAT1 is induced by ER $\alpha$ and the differentiation program involving GATA3 and TJP1 is inhibited; the feedback loop goes forward. When differentiation or the maintenance of the differentiation state of the mammary epithelium is favored, the growth signaling instigated by ER $\alpha$ has to be attenuated or stopped, and the differentiation factors such as GATA3 need to be upregulated; the feedback loop goes the other direction. It is intriguing to even speculate that the balance of the feedback loop is essential for normal mammary physiology, and tilted balance of this loop contributes to the development and progression of breast cancer.

In summary, our current study revealed that NEAT1 is a facultative component of the SIN3A complex, shedding new light on the understanding of the mechanistic actions of NEAT1 and the SIN3A complex. Our experiments identified the ER $\alpha$-NEAT1-FOXN3/ NEAT1/SIN3A-GATA3 axis that is implicated in breast cancer metastasis, providing a molecular basis for the understanding of the pathophysiological function of FOXN3 and supporting the pursuit of these molecules as potential targets for breast cancer intervention.

\section{Methods}

Antibodies and reagents. Antibodies used were $\alpha \mathrm{ER} \alpha$ (sc-8002), $\alpha \mathrm{HDAC} 1$ (sc-7872), $\alpha$ HDAC2 (sc-7899), and $\alpha \mathrm{RbAp} 46 / 48$ (sc-373873) from Santa Cruz Biotechnology; $\alpha$ GATA3 (GTX30600), $\alpha$ SIN3A (GTX129156), and $\alpha \mathrm{TJP1}$ (GTX108592) from GeneTex; $\alpha \mathrm{CDH} 1$ (orb213705) and $\alpha \mathrm{FOXN} 3$ (orb182590) from Biorbyt; $\alpha \mathrm{H} 3$ (ab1791), $\alpha$ FOXN3 (ab129453), $\alpha \mathrm{SAP} 18$ (ab31748), and $\alpha$ SAP130 (ab114978) from Abcam; $\alpha$ His (AE003) from Abclonal; $\alpha$ SAP180 (24499-1-AP), $\alpha$ HDAC1 (66085-1-Ig), and $\alpha-\gamma$ catenin (1146-1-1AP) from Proteintech; $\alpha \mathrm{H} 3 \mathrm{Ac}$ (61637) and $\alpha \mathrm{SIN} 3 \mathrm{~A}$ (39865) from Active Motif; $\alpha$ - $\alpha$-catenin (3240S) and $\alpha$-vimentin (5741P) from Cell Signaling Technology; and $\alpha$ FLAG (F3165) and $\alpha$-fibronectin (F7387) from Sigma-Aldrich. The specificities of the bands in Western blots were controlled according to the commercial antibodies' instructions. Control siRNA and siRNAs for FOXN3, MALAT1, and NEAT1 as well as shRNAs for FOXN3, NEAT1, SIN3A, GATA3, and SAP18 were synthesized by Shanghai GenePharma. Protein A/G Sepharose CL-4B beads were from Amersham Biosciences, and protease inhibitor cocktail was from Roche Applied Science.

Cell culture and transfection. Cell lines used were obtained from the American Type Culture Collection (ATCC). MCF-7 cells were maintained in DMEM supplemented with $10 \%$ FBS in a humidified incubator equilibrated with $5 \% \mathrm{CO}_{2}$ at $37^{\circ} \mathrm{C}$. MDA-MB-231 cells were cultured in L-15 medium supplemented with $10 \% \mathrm{FBS}$ without $\mathrm{CO}_{2}$. Transfections were carried out using Lipofectamine 2000 or Lipofectamine RNAiMAX Reagent (Invitrogen) according to the manufacturer's instruction. Each experiment was performed in triplicate and repeated at least 3 times. For the RNAi experiment, at least 3 independent siRNA/shRNA sequences were tested for each gene, and the one with the best efficiency was used. The siRNA sequences are provided in Supplemental Table 4.

Reporter assay. Luciferase activity was measured using the DualLuciferase kit (Promega) according to the manufacturer's protocol. Each experiment was performed in triplicate and repeated at least 3 times.
Silver staining and mass spectrometry. Cellular extracts from MCF7 cells expressing FLAG-FOXN3 were prepared and applied to antiFLAG M2 affinity gel (Sigma-Aldrich) following the manufacturer's protocol. FLAG peptide $(0.2 \mathrm{mg} / \mathrm{ml}$; Sigma-Aldrich) was added to the column to elute protein complex. Fractions of the bed volume were collected and resolved on SDS-PAGE and silver-stained, and bands were excised and subjected to liquid chromatography-tandem mass spectrometry sequencing and data analysis.

FPLC, IP, and Western blotting. Fast protein liquid chromatography (FPLC), IP, and Western blotting were performed according to the procedures described previously $(48,49)$.

iRIP-Seq. MCF-7 cells were cross-linked on ice with UV irradiation type C $(254 \mathrm{~nm})$ at $400 \mathrm{~mJ} / \mathrm{cm}^{2}$ in the presence of cold PBS. Cells were collected and pelleted at $1,000 \mathrm{~g}$ at $4^{\circ} \mathrm{C}$, and lysed in cold wash buffer ( $1 \times$ PBS, $0.1 \%$ SDS, $0.5 \%$ NP-40, and 0.5\% sodium deoxycholate) containing $200 \mathrm{U} / \mathrm{ml}$ RNase inhibitor (Takara) and protease inhibitor cocktail (Roche) on ice for 10 minutes. Cell lysates were centrifuged at $16,000 \mathrm{~g}$ for 20 minutes at $4^{\circ} \mathrm{C}$ followed by addition of RQI (Promega) to a final concentration of $1 \mathrm{U} / \mu \mathrm{l}$ and incubation in a water bath for 5 minutes at $37^{\circ} \mathrm{C}$ and then cooled down for 5 minutes on ice. For IP, the supernatant was incubated with $15 \mu \mathrm{g}$ antibodies or control IgG overnight at $4^{\circ} \mathrm{C}$. The immunoprecipitates were further incubated with protein $\mathrm{A}$ or $\mathrm{G}$ Dynabeads for 3 hours at $4^{\circ} \mathrm{C}$. After application to a magnet and removal of the supernatants, the beads were sequentially washed with lysis buffer, high-salt buffer (250 mM Tris 7.4, 750 $\mathrm{mM} \mathrm{NaCl}, 10 \mathrm{mM}$ EDTA, 0.1\% SDS, 0.5\% NP-40, and 0.5 deoxycholate), and polynucleotide kinase (PNK) buffer (50 mM Tris, 20 mM EGTA, and 0.5\% NP-40) twice. After addition of 1:1,000,000 MNase (300 U/ $\mu \mathrm{l}$; Fermentas), beads were resuspended, incubated in a water bath for 15 minutes at $37^{\circ} \mathrm{C}$, and applied to magnet to remove supernatants. The beads were then washed with PNK buffer twice and resuspended in 1 volume of dephosphorylation buffer followed by addition of calf intestinal alkaline phosphatase (New England Biolabs) to a final concentration of $0.5 \mathrm{U} / \mu \mathrm{l}$ and incubation for $10 \mathrm{~min}$ utes at $37^{\circ} \mathrm{C}$. The beads were then washed twice in $1 \mathrm{ml}$ of PNK buffer without DTT. The beads were subsequently resuspended in 1 original bead volume of PNK buffer and added with ATP to a final concentration of $100 \mu \mathrm{M}$ and T4 PNK (New England Biolabs) to $1 \mathrm{U} / \mu \mathrm{l}$. The magnetic bead suspension was incubated at $37^{\circ} \mathrm{C}$ for 30 minutes and washed 3 times with $800 \mu$ of PNK buffer without DTT followed by resuspension in $100 \mu \mathrm{l}$ of SDS+DTT elution buffer and incubation in a heat block at $70^{\circ} \mathrm{C}$ for 20 minutes to denature and release the crosslinked RNAs. The magnetic beads were removed on a separator, and the supernatant was transferred to a clean $1.5-\mathrm{ml}$ microfuge tube, followed by addition of an equal volume of $2 \times$ proteinase $\mathrm{K}$ buffer and proteinase $\mathrm{K}$ (Roche) to a final concentration of $1.2 \mathrm{mg} / \mathrm{ml}$ and incubation at $55^{\circ} \mathrm{C}$ for 60 minutes. RNAs were recovered by acidic phenol/chloroform/isoamylalcohol extraction (25:24:1, pH 4.0) followed by chloroform extraction. One microliter of glycogen $(10 \mathrm{mg} /$ $\mathrm{ml}$ stock) was then added, and RNAs were precipitated by 3 volumes of ethanol and dissolved in $10.5 \mu \mathrm{l}$ water. The recovered RNAs were used to generate a paired-end sequencing library with TruSeq small RNA library preparation kit (Illumina) following the manufacturer's instruction. Libraries were purified, quantified, and stored at $-80^{\circ} \mathrm{C}$ until used for sequencing. For high-throughput sequencing, the libraries were applied to Illumina NextSeq 500 system for 150-nt paired-end sequencing by ABLife Inc. 
LncRNA microarray. Total RNAs containing noncoding RNAs were extracted from MCF-7 cells and MDA-MB-231 cells with Trizol reagent (Life Technologies) for reverse transcription in the presence of Cy3-dCTP (GE Healthcare). Amplified/Cy3-labeled cDNAs were hybridized onto oligonucleotide microarrays containing 40,916 probes of lncRNAs (CapitalBio Human lncRNA 4.0 Array). Data were analyzed using GeneSpring software version 13.0 (Agilent).

RNA immunoprecipitation. Cell lysates were diluted in RIP buffer (50 mM Tris, $\mathrm{pH} 7.4,200 \mathrm{mM} \mathrm{NaCl}, 0.5 \% \mathrm{NP}-40,0.5 \%$ sodium deoxycholate, 10\% glycerol, $1 \mathrm{mM}$ DTT, $1 \mathrm{mM}$ PMSF, 1:500 protease inhibitor cocktail, 1:100 RNase inhibitor). After addition of $40 \mathrm{U}$ RQ1 DNase to digest DNA and incubation at $37^{\circ} \mathrm{C}$ for 10 minutes, cell lysates were cooled down on ice, cleared by centrifugation at $20,000 \mathrm{~g}$ for 10 minutes, and incubated with antibody for 4 hours at $4^{\circ} \mathrm{C}$. Immunocomplexes were recovered with protein A-coupled Dynabeads (Invitrogen) for 2 hours at $4^{\circ} \mathrm{C}$ that were washed in RIP-W buffer ( $20 \mathrm{mM}$ Tris-Cl, pH 7.4, 2,000 $\mathrm{mM} \mathrm{NaCl}, 1 \mathrm{mM}$ EDTA, 0.3 Triton X-100, 5\% glycerol, 10-20 $\mu \mathrm{g}$ freshly added yeast transfer RNA per ml) 4 times at $4^{\circ} \mathrm{C}$. RNAs were eluted and extracted with TRIzol (Invitrogen).

RNA pull-down assay. NEAT1 fragment (1-500 nt) was generated by in vitro transcription using PCR-amplified template DNA from pcDNA3.1-NEAT1 and biotinylated. For in vitro binding assays, $2 \mu \mathrm{g}$ of GST/His double-tagged FOXN3, SIN3A, or SAP18 was incubated with $0.5 \mu \mathrm{g}$ NEAT1 fragments bound to streptavidin-conjugated agarose resin for 30 minutes at room temperature, washed in RIP buffer 4 times at room temperature, and resuspended in $30 \mathrm{ml}$ of $2^{\prime}$ SDS-PAGE loading buffer. Bound proteins were detected by Western blotting.

Real-time quantitative reverse transcription PCR. Real-time quantitative reverse transcription PCR (qPCR) was performed essentially the same as previously described $(48,49)$. The primers are provided in Supplemental Table 5.

ChIP-Seq. Approximately $5 \times 10^{7}$ cells were used for each ChIP-Seq assay. Chromatin DNAs precipitated by polyclonal antibodies against SIN3A or FOXN3 were purified with the Qiagen PCR purification kit. Indepth whole-genome DNA sequencing was performed by BGI (Shenzhen, China). The raw sequencing image data were examined by the Illumina analysis pipeline, aligned to the unmasked human reference genome (UCSC GRCh37, hg19) using Bowtie 2, and further analyzed by MACS (Model-based Analysis for ChIP-Seq; https://github.com/taoliu/ MACS). Enriched binding peaks were generated after filtering through control input. Genomic distribution of FOXN3 or SIN3A binding sites was analyzed by ChIPseeker, an R package for ChIP peak annotation, comparison, and visualization. De novo motif screening was performed on sequences \pm 100 bp from the centers of FOXN3 or SIN3A binding peaks based on the MEME suite (http://meme-suite.org/).

ChIP, qChIP, and Re-ChIP. ChIP, qChIP, and Re-ChIP were performed essentially the same as previously described $(48,49)$. The primers are provided in Supplemental Table 5.

Transwell cell invasion assay. Transwell cell invasion assays were performed essentially the same as previously described $(48,49)$.

Lentivirus production and infection. Recombinant lentiviruses expressing shFOXN3, shNEAT1, shSIN3A, shSAP18, shGATA3, FLAGFOXN3, or NEAT1 were constructed by Shanghai GenePharma. Concentrated viruses were used to infect $5 \times 10^{5}$ cells in a $60-\mathrm{mm}$ dish with $8 \mu \mathrm{g} / \mathrm{ml}$ Polybrene. Infected MCF-7 cells were then subjected to sorting target expression. The shRNA sequences used are provided in Supplemental Table 4.
In vivo metastasis. MCF-7 cells that had been engineered to stably express firefly luciferase (PerkinElmer) were infected with lentiviruses carrying vector+shSCR, FOXN3+shSCR, FOXN3+shSIN3A, FOXN3+GATA3, NEAT1+shSCR, or NEAT1+shSIN3A. These cells were inoculated into the left abdominal mammary fat pad of 6-week-old ovariectomized female SCID mice implanted with E2 pellets. For bioluminescence imaging, mice were injected with $200 \mathrm{mg} / \mathrm{g}$ of D-luciferin in PBS abdominally. Fifteen minutes after injection, mice were anesthetized and bioluminescence was imaged with a charge-coupled device camera (IVIS; Xenogen). Bioluminescence images were obtained with a 15 -cm field of view, binning (resolution) factor of $8,1 /$ f stop, open filter, and an imaging time of 30 seconds to 2 minutes. Bioluminescence from relative optical intensity was defined manually. Photon flux was normalized to background, which was defined from a relative optical intensity drawn over a mouse not given an injection of luciferin.

Tissue specimens. Breast carcinomas and the adjacent normal mammary tissues were obtained from surgical specimens from patients with breast cancer. Samples were selected from patients for whom the complete clinicopathological information was available. Samples were frozen in liquid nitrogen immediately after surgical removal and maintained at $-80^{\circ} \mathrm{C}$ until mRNA extraction. Breast tissue arrays were prepared and subjected to immunohistochemical analysis with standard DAB staining protocols.

Statistics. Results were reported as mean \pm SD for triplicate experiments unless otherwise noted. SPSS version 17.0, 2-tailed $t$ test, and 1-way or 2-way ANOVA were used as indicated in the legends. The correlation coefficients were calculated by R programming. A $P$ value less than 0.05 was considered significant. Breast tumor data sets were downloaded from GEO (http://www.ncbi.nlm.nih.gov/geo). Data for Kaplan-Meier survival analysis were from Kaplan-Meier Plotter (http://kmplot.com/analysis/index.php?p=service\&cancer=breast).

Accession number. ChIP-Seq, iRIP-Seq, and microarray data were deposited at the Gene Expression Omnibus (GEO) database (http:// www.ncbi.nlm.nih.gov/geo/) with accession number GSE93781.

Study approval. All studies were approved by the Ethics Committee of Peking University Health Science Center, and informed consent was obtained from all patients. Animal handling and procedures were approved by Peking University Health Science Center Institutional Animal Care.

\section{Author contributions}

WL and YS conceived the project, designed experiments, analyzed data, and wrote the manuscript. WL, ZZ, XL, XC, Yi Zhang, $\mathrm{XH}$, Yu Zhang, SL, JY, BX, LH, LS, and JL performed experiments and analyzed data.

\section{Acknowledgments}

This work was supported by grants (91219201 and 81530073 to YS, and 81572771 to LJ) from the National Natural Science Foundation of China, and grants (2016YFC1302304 to YS and 2014CB542004 to JL) from the Ministry of Science and Technology of China.

Address correspondence to: Yongfeng Shang, Department of Biochemistry and Molecular Biology, Peking University Health Science Center, 38 Xueyuan Road, Beijing 100191, China. Phone: 86.10.82805118; Email: yshang@hsc.pku.edu.cn. 
1. Kaestner KH, Knochel W, Martinez DE. Unified nomenclature for the winged helix/forkhead transcription factors. Genes Dev. 2000;14(2):142-146.

2. Lehmann OJ, Sowden JC, Carlsson P, Jordan T, Bhattacharya SS. Fox's in development and disease. Trends Genet. 2003;19(6):339-344.

3. Schuff M, Rössner A, Wacker SA, Donow C, Gessert S, Knöchel W. FoxN3 is required for craniofacial and eye development of Xenopus laevis. Dev Dyn. 2007;236(1):226-239.

4. Samaan $\mathrm{G}$, et al. Foxn3 is essential for craniofacial development in mice and a putative candidate involved in human congenital craniofacial defects. Biochem Biophys Res Commun. 2010;400(1):60-65

5. Pati D, Keller C, Groudine M, Plon SE. Reconstitution of a MEC1-independent checkpoint in yeast by expression of a novel human fork head cDNA. Mol Cell Biol. 1997;17(6):3037-3046.

6. Huot $\mathrm{G}$, et al. CHES1/FOXN3 regulates cell proliferation by repressing PIM2 and protein biosynthesis. Mol Biol Cell. 2014;25(5):554-565.

7. Sun J, et al. The transcription factor FOXN3 inhibits cell proliferation by downregulating E2F5 expression in hepatocellular carcinoma cells. Oncotarget. 2016;7(28):43534-43545.

8. Scott KL, Plon SE. Loss of Sin3/Rpd3 histone deacetylase restores the DNA damage response in checkpoint-deficient strains of Saccharomyces cerevisiae. Mol Cell Biol. 2003;23(13):4522-4531.

9. Busygina V, Kottemann MC, Scott KL, Plon SE, Bale AE. Multiple endocrine neoplasia type 1 interacts with forkhead transcription factor CHES1 in DNA damage response. Cancer Res. 2006;66(17):8397-8403.

10. Scott KL, Plon SE. CHES1/FOXN3 interacts with Ski-interacting protein and acts as a transcriptional repressor. Gene. 2005;359:119-126.

11. Yu J, et al. CHES-1-like, the ortholog of a nonobstructive azoospermia-associated gene, blocks germline stem cell differentiation by upregulating Dpp expression in Drosophila testis. Oncotarget. 2016;7(27):42303-42313

12. Chang JT, et al. Identification of differentially expressed genes in oral squamous cell carcinoma (OSCC): overexpression of NPM, CDK1 and NDRG1 and underexpression of CHES1. Int J Cancer. 2005;114(6):942-949.

13. Markowski J, et al. Gene expression profile analysis in laryngeal cancer by high-density oligonucleotide microarrays. J Physiol Pharmacol. 2009;60(suppl 1):57-63.

14. Basso K, Margolin AA, Stolovitzky G, Klein U, Dalla-Favera R, Califano A. Reverse engineering of regulatory networks in human B cells. Nat Genet. 2005;37(4):382-390.

15. Robertson E, Perry C, Doherty R, Madhusudan S. Transcriptomic profiling of Forkhead box transcription factors in adult glioblastoma multiforme. Cancer Genomics Proteomics. 2015;12(3):103-112.
16. Nagel S, Meyer C, Kaufmann M, Drexler HG, MacLeod RA. Deregulated FOX genes in Hodgkin lymphoma. Genes Chromosomes Cancer. 2014;53(11):917-933 .

17. Sawiris GP, Sherman-Baust CA, Becker KG, Cheadle C, Teichberg D, Morin PJ. Development of a highly specialized cDNA array for the study and diagnosis of epithelial ovarian cancer. Cancer Res. 2002;62(10):2923-2928.

18. Iżykowska K, et al. Submicroscopic genomic rearrangements change gene expression in T-cel large granular lymphocyte leukemia. Eur J Haematol. 2014;93(2):143-149.

19. Guttman M, et al. Chromatin signature reveals over a thousand highly conserved large non-coding RNAs in mammals. Nature. 2009;458(7235):223-227.

20. Bonasio R, Shiekhattar R. Regulation of transcription by long noncoding RNAs. Annu Rev Genet. 2014;48:433-455.

21. Rinn JL, Chang HY. Genome regulation by long noncoding RNAs. Annu Rev Biochem. 2012;81:145-166.

22. Clemson CM, et al. An architectural role for a nuclear noncoding RNA: NEAT1 RNA is essential for the structure of paraspeckles. Mol Cell. 2009;33(6):717-726.

23. Hirose $\mathrm{T}$, et al. NEAT1 long noncoding RNA regulates transcription via protein sequestration within subnuclear bodies. Mol Biol Cell. 2014;25(1):169-183.

24. West JA, et al. The long noncoding RNAs NEAT1 and MALAT 1 bind active chromatin sites. Mol Cell. 2014;55(5):791-802.

25. Chakravarty D, et al. The oestrogen receptor $\alpha$-regulated lncRNA NEAT1 is a critical modulator of prostate cancer. Nat Commun. 2014;5:5383.

26. Chai Y, Liu J, Zhang Z, Liu L. HuR-regulated IncRNA NEAT1 stability in tumorigenesis and progression of ovarian cancer. Cancer Med. 2016;5(7):1588-1598.

27. Fujimoto A, et al. Whole-genome mutational landscape and characterization of noncoding and structural mutations in liver cancer. Nat Genet. 2016;48(5):500-509.

28. Adriaens C, et al. p53 induces formation of NEAT1 IncRNA-containing paraspeckles that modulate replication stress response and chemosensitivity. Nat Med.2016;22(8):861-868.

29. Wang P, et al. Long noncoding RNA NEAT1 promotes laryngeal squamous cell cancer through regulating miR-107/CDK6 pathway. J Exp Clin Cancer Res. 2016;35:22.

30. Sun C, et al. Long non-coding RNA NEAT1 promotes non-small cell lung cancer progression through regulation of miR-377-3p-E2F3 pathway. Oncotarget. 2016;7(32):51784-51814.

31. Zhen L, Yun-Hui L, Hong-Yu D, Jun M, Yi-Long Y. Long noncoding RNA NEAT1 promotes glioma pathogenesis by regulating miR-449b-5p/c-Met axis. Tumour Biol. 2016;37(1):673-683.

32. Kaneko S, et al. Interactions between JARID2 and noncoding RNAs regulate PRC2 recruitment to chromatin. Mol Cell. 2014;53(2):290-300.

33. Tsai MC, et al. Long noncoding RNA as modular scaffold of histone modification complexes. Science. 2010;329(5992):689-693.

34. Yao H, Brick K, Evrard Y, Xiao T, Camerini-Otero RD, Felsenfeld G. Mediation of CTCF transcriptional insulation by DEAD-box RNA-binding protein $\mathrm{p} 68$ and steroid receptor RNA activator SRA. Genes Dev. 2010;24(22):2543-2555.

35. Vance KW, Ponting CP. Transcriptional regulatory functions of nuclear long noncoding RNAs. Trends Genet. 2014;30(8):348-355.

36. Hanahan D, Weinberg RA. Hallmarks of cancer the next generation. Cell.2011;144(5):646-674.

37. Kouros-Mehr H, Slorach EM, Sternlicht MD, Werb Z. GATA-3 maintains the differentiation of the luminal cell fate in the mammary gland. Cell. 2006;127(5):1041-1055.

38. Yoon NK, et al. Higher levels of GATA3 predict better survival in women with breast cancer. Hum Pathol. 2010;41(12):1794-1801.

39. Shen L, Shao N, Liu X, Nestler E. ngs.plot: Quick mining and visualization of next-generation sequencing data by integrating genomic databases. BMC Genomics. 2014;15:284.

40. Spriggs RV, Jones $\mathrm{S}$. RNA-binding residues in sequence space: conservation and interaction patterns. Comput Biol Chem. 2009;33(5):397-403.

41. Baltz AG, et al. The mRNA-bound proteome and its global occupancy profile on protein-coding transcripts. Mol Cell. 2012;46(5):674-690.

42. Nakagawa S, Hirose T. Paraspeckle nuclear bodies--useful uselessness? Cell Mol Life Sci. 2012;69(18):3027-3036.

43. Naganuma T, Hirose T. Paraspeckle formation during the biogenesis of long non-coding RNAs. RNA Biol. 2013;10(3):456-461.

44. Kadamb R, Mittal S, Bansal N, Batra H, Saluja D. Sin3: insight into its transcription regulatory functions. Eur JCell Biol. 2013;92(8-9):237-246.

45. Asselin-Labat ML, et al. Gata-3 is an essential regulator of mammary-gland morphogenesis and luminal-cell differentiation. Nat Cell Biol. 2007;9(2):201-209.

46. Liang J, Shang Y. Estrogen and cancer. Annu Rev Physiol. 2013;75:225-240.

47. Yu QC, Verheyen EM, Zeng YA. Mammary development and breast cancer: a Wnt perspective. Cancers (Basel). 2016;8(7):E65

48. Shan L, et al. FOXK2 elicits massive transcription repression and suppresses the hypoxic response and breast cancer carcinogenesis. Cancer Cell. 2016;30(5):708-722.

49. Si W, et al. Dysfunction of the reciprocal feedback loop between GATA3- and ZEB2-nucleated repression programs contributes to breast cance metastasis. Cancer Cell. 2015;27(6):822-836. 